\title{
Adaptive Control Based on Retrospective Cost Optimization
}

\author{
Mario A. Santillo* and Dennis S. Bernstein \\ University of Michigan, Ann Arbor, Michigan 48109
}

DOI: $10.2514 / 1.46741$

\begin{abstract}
We present a discrete-time adaptive control law for stabilization, command-following, and disturbance rejection that is effective for systems that are unstable, multi-input/multi-output, and/or non-minimum phase. The adaptive control algorithm includes guidelines concerning the modeling information needed for implementation. This information includes the relative degree, the first nonzero Markov parameter, and the non-minimum-phase zeros. Except when the plant has non-minimum-phase zeros whose absolute value is less than the plant's spectral radius, the required zero information can be approximated by a sufficient number of Markov parameters. No additional information about the poles or zeros need be known. We present numerical examples to illustrate the algorithm's effectiveness in handling systems with errors in the required modeling data, unknown latency, sensor noise, and saturation.
\end{abstract}

\section{Introduction}

$\mathbf{U}$ NLIKE robust control, which chooses control gains based on a prior, fixed level of modeling uncertainty, adaptive control algorithms tune the feedback gains in response to the true plant and exogenous signals: that is, commands and disturbances. Generally speaking, adaptive controllers require less prior modeling information than robust controllers and thus can be viewed as highly parameter-robust control laws. The price paid for the ability of adaptive control laws to operate with limited prior modeling information is the complexity of analyzing and quantifying the stability and performance of the closed-loop system, especially in light of the fact that adaptive control laws, even for linear plants, are nonlinear.

Stability and performance analysis of adaptive control laws often entails assumptions on the dynamics of the plant. For example, a widely invoked assumption in adaptive control is passivity [1], which is restrictive and difficult to verify in practice. A related assumption is that the plant is minimum phase $[2,3]$, which may entail the same difficulties. In fact, sampling may give rise to non-minimum-phase zeros whether or not the continuous-time system is minimum phase [4], which must ultimately be accounted for by any adaptive control algorithm implemented digitally in a sampled-data control system. Beyond these assumptions, adaptive control laws are known to be sensitive to unmodeled dynamics and sensor noise [5,6], which necessitates robust adaptive control laws [7].

In addition to these basic issues, adaptive control laws may entail unacceptable transients during adaptation, which may be exacerbated by actuator limitations [8-10]. In fact, adaptive control under extremely limited modeling information, such as uncertainty in the sign of the high-frequency gain $[11,12]$, may yield a transient response that exceeds the practical limits of the plant. Therefore, the type and quality of the available modeling information as well as the speed of adaptation must be considered in the analysis and implementation of adaptive control laws. These issues are stressed in [13].

Adaptive control laws have been developed in both continuoustime and discrete-time settings. In the present paper, we consider discrete-time adaptive control laws, since these control laws can be implemented directly in embedded code for sampled-data control

Received 15 August 2009; revision received 7 November 2009; accepted for publication 16 November 2009. Copyright (C) 2009 by Dennis S. Bernstein. Published by the American Institute of Aeronautics and Astronautics, Inc., with permission. Copies of this paper may be made for personal or internal use, on condition that the copier pay the $\$ 10.00$ per-copy fee to the Copyright Clearance Center, Inc., 222 Rosewood Drive, Danvers, MA 01923; include the code 0731-5090/10 and \$10.00 in correspondence with the CCC.

*National Defense Science and Engineering Graduate Fellow, Aerospace Engineering Department, 1320 Beal Avenue. Member AIAA.

${ }^{\dagger}$ Professor, Aerospace Engineering Department, 1320 Beal Avenue. systems without requiring an intermediate discretization step that may entail loss of stability margins.

References on discrete-time adaptive control include [2,3,14-24]. In [2], a discrete-time adaptive control law with guaranteed stability is developed under a minimum-phase assumption. Extensions given in [3] based on internal model control [25] and Lyapunov analysis also invoke this assumption. To circumvent the minimum-phase assumption, the zero annihilation periodic control law [23] uses lifting to move all of the plant zeros to the origin. The drawback of lifting, however, is the need for open-loop operation during alternating data windows. An alternative approach, developed in $[14,15,17,18]$, is to exploit knowledge of the non-minimum-phase zeros. In [14], knowledge of the non-minimum-phase zeros is used to allow matching of a desired closed-loop transfer function, recognizing that minimum-phase zeros can be canceled but not moved, whereas non-minimum-phase zeros can neither be canceled nor moved. In $[15,18]$, knowledge of a diagonal matrix that contains the non-minimum-phase zeros is used within a multi-input/multioutput (MIMO) direct adaptive control algorithm. Finally, knowledge of the unstable zeros of a rapidly sampled continuous-time singleinput/single-output (SISO) system with a real non-minimum-phase zero is used in [17].

Motivated by the adaptive control laws given in $[3,24]$, the goal of the present paper is to develop a discrete-time adaptive control law that is effective for non-minimum-phase systems. In particular, we present an adaptive control algorithm that extends the retrospective cost optimization approach used in [24]. This extension is based on a retrospective cost that includes control weighting as well as a learning rate, which can be used to adjust the rate of controller convergence and thus the transient behavior of the closed-loop system. Unlike [24], which uses a gradient update, the present paper uses a Newtonlike update for the controller gains, as the closed-form solution to a quadratic optimization problem. No offline calculations are needed to implement the algorithm. A key aspect of this extension is the fact that the required modeling information is the relative degree, the first nonzero Markov parameter, and non-minimum-phase zeros, if any. Except when the plant has non-minimum-phase zeros whose absolute value is less than the plant's spectral radius, we show that the required zero information can be approximated by a sufficient number of Markov parameters from the control inputs to the performance variables. No matching conditions are required on either the plant uncertainty or disturbances.

The goal of the present paper is to develop the retrospective correction filter (RCF) adaptive control algorithm and demonstrate its effectiveness for handling non-minimum-phase zeros. To this end, we consider a sequence of examples of increasing complexity, ranging from SISO minimum-phase plants to MIMO non-minimumphase plants, including stable and unstable cases. We then revisit these plants under offnominal conditions: that is, with uncertainty in the required plant modeling data, unknown latency, sensor noise, and 
saturation. These numerical examples provide guidance into choosing the design parameters of the adaptive control law in terms of the learning rate, data window size, controller order, modeling data, and control weightings. Preliminary versions of the present paper are given in [26,27].

\section{Problem Formulation}

Consider the MIMO discrete-time system

$$
\begin{gathered}
x(k+1)=A x(k)+B u(k)+D_{1} w(k) \\
y(k)=C x(k)+D_{2} w(k) \\
z(k)=E_{1} x(k)+E_{0} w(k)
\end{gathered}
$$

where $x(k) \in \mathbb{R}^{n}, y(k) \in \mathbb{R}^{l_{y}}, z(k) \in \mathbb{R}^{l_{z}}, u(k) \in \mathbb{R}^{l_{u}}, w(k) \in \mathbb{R}^{l_{w}}$, and $k \geq 0$. Our goal is to develop an adaptive output-feedback controller under which the performance variable $z$ is minimized in the presence of the exogenous signal $w$. In Eqs. (1-3), $w$ can represent either a command signal to be followed, an external disturbance to be
Letting the data window size $p$ be a positive integer, we define the extended performance vector $Z(k) \in \mathbb{R}^{p l_{z}}$ and $U_{1}(k) \in \mathbb{R}^{q_{c} l_{u}}$ by

$$
Z(k) \triangleq\left[\begin{array}{c}
z(k) \\
\vdots \\
z(k-p+1)
\end{array}\right], \quad U_{1}(k) \triangleq\left[\begin{array}{c}
u(k-1) \\
\vdots \\
u\left(k-q_{c}\right)
\end{array}\right]
$$

where $q_{c} \triangleq n+p-1$. The data window size $p$ has a small but noticeable effect on transient behavior. Now Eq. (4) can be written in the form

$$
Z(k)=W_{z w} \phi_{z w}(k)+B_{z u} U_{1}(k)
$$

where $\quad W_{z w} \in \mathbb{R}^{p l_{z} \times\left[q_{c} l_{z}+\left(q_{c}+1\right) l_{w}\right]}, \quad B_{z u} \in \mathbb{R}^{p l_{z} \times q_{c} l_{u}}, \quad$ and $\quad \phi_{z w} \in$ $\mathbb{R}^{q_{c} l_{z}+\left(q_{c}+1\right) l_{w}}$ are given by

$$
W_{z w} \triangleq\left[\begin{array}{cccccccccccc}
-\alpha_{1} I_{l_{z}} & \cdots & -\alpha_{n} I_{l_{z}} & 0_{l_{z} \times l_{z}} & \cdots & 0_{l_{z} \times l_{z}} & \gamma 0 & \cdots & \gamma n & 0_{l_{z} \times l_{w}} & \cdots & 0_{l_{z} \times l_{w}} \\
0_{l_{z} \times l_{z}} & \ddots & & \ddots & \ddots & \vdots & 0_{l_{z} \times l_{w}} & \ddots & & \ddots & \ddots & \vdots \\
\vdots & \ddots & \ddots & & \ddots & 0_{l_{z} \times l_{z}} & \vdots & \ddots & \ddots & & \ddots & 0_{l_{z} \times l_{w}} \\
0_{l_{z} \times l_{z}} & \cdots & 0_{l_{z} \times l_{z}} & -\alpha_{1} I_{l_{z}} & \cdots & -\alpha_{n} I_{l_{z}} & 0_{l_{z} \times l_{w}} & \cdots & 0_{l_{z} \times l_{w}} & \gamma 0 & \cdots & \gamma n
\end{array}\right]
$$

rejected, or both. For example, if $D_{1}=0$ and $E_{0} \neq 0$, then the objective is to have the output $E_{1} x$ follow the command signal $-E_{0} w$. On the other hand, if $D_{1} \neq 0$ and $E_{0}=0$, then the objective is to reject the disturbance $w$ from the performance variable $E_{1} x$. The combined command-following and disturbance-rejection problem is addressed when $D_{1}$ and $E_{0}$ are suitably partitioned matrices. More precisely, if $D_{1}=\left[\begin{array}{ll}D_{11} & 0\end{array}\right], E_{0}=\left[\begin{array}{ll}0 & E_{02}\end{array}\right]$, and

$$
w(k)=\left[\begin{array}{l}
w_{1}(k) \\
w_{2}(k)
\end{array}\right]
$$

then the objective is to have $E_{1} x$ follow the command $-E_{02} w_{2}$ while rejecting the disturbance $D_{11} w_{1}$. Finally, if $D_{1}$ and $E_{0}$ are zero matrices, then the objective is output stabilization, that is, convergence of $z$ to zero. We assume that $(A, B)$ is stabilizable, $(A, C)$ and $\left(A, E_{1}\right)$ are detectable, and that measurements of $y$ and $z$ are available for feedback. If the command signal is included as a component of $y$, then the adaptive controller has a feedforward architecture. For disturbance-rejection problems, the controller does not require measurements of the external disturbance $w$.

\section{ARMAX Modeling}

Consider the ARMAX representation of Eqs. (1) and (3), given by

$$
z(k)=\sum_{i=1}^{n}-\alpha_{i} z(k-i)+\sum_{i=1}^{n} \beta_{i} u(k-i)+\sum_{i=0}^{n} \gamma_{i} w(k-i)
$$

where $\alpha_{1}, \ldots, \alpha_{n} \in \mathbb{R}, \beta_{1}, \ldots, \beta_{n} \in \mathbb{R}^{l_{z} \times l_{u}}$, and $\gamma_{0}, \ldots, \gamma_{n} \in \mathbb{R}^{l_{z} \times l_{w}}$. We define the relative degree $d \geq 1$ as the smallest positive integer $i$ such that the $i$ th Markov parameter $H_{i} \triangleq E_{1} A^{i-1} B \in \mathbb{R}^{l_{z} \times l_{u}}$ is nonzero. Note that if $d=1$, then $H_{1}=\beta_{1}$, whereas if $d \geq 2$, then

$$
\beta_{1}=\cdots=\beta_{d-1}=H_{1}=\cdots=H_{d-1}=0
$$

$$
B_{z u} \triangleq\left[\begin{array}{cccccc}
\beta_{1} & \cdots & \beta_{n} & 0_{l_{z} \times l_{u}} & \cdots & 0_{l_{z} \times l_{u}} \\
0_{l_{z} \times l_{u}} & \ddots & & \ddots & \ddots & \vdots \\
\vdots & \ddots & \ddots & & \ddots & 0_{l_{z} \times l_{u}} \\
0_{l_{z} \times l_{u}} & \cdots & 0_{l_{z} \times l_{u}} & \beta_{1} & \cdots & \beta_{n}
\end{array}\right]
$$

and

$$
\phi_{z w}(k) \triangleq\left[\begin{array}{c}
z(k-1) \\
\vdots \\
z(k-p-n+1) \\
w(k) \\
\vdots \\
w(k-p-n+1)
\end{array}\right]
$$

Note that $W_{z w}$ includes modeling information about the plant poles and exogenous input path, whereas $B_{z u}$ includes modeling information about the plant zeros. Both $W_{z w}$ and $B_{z u}$ have block-Toeplitz structure.

\section{Controller Construction}

To formulate an adaptive control algorithm for Eqs. (1 $\underline{-}-\underline{3})$, we use a strictly proper time-series controller of order $n_{c}$ such that the control $u(k)$ is given by

$$
u(k)=\sum_{i=1}^{n_{c}} P_{i}(k) u(k-i)+\sum_{i=1}^{n_{c}} Q_{i}(k) y(k-i)
$$

where $P_{i}(k) \in \mathbb{R}^{l_{u} \times l_{u}}$ and $Q_{i}(k) \in \mathbb{R}^{l_{u} \times l_{y}}$ for all $i=1, \ldots, n_{c}$. The controller order $n_{c}$ is determined by standard control guidelines in terms of stabilization and disturbance rejection. The control (10) can be expressed as 
where

$$
\begin{aligned}
& \theta(k) \triangleq\left[\begin{array}{llllll}
Q_{1}(k) & \cdots & Q_{n_{c}}(k) & P_{1}(k) & \cdots & P_{n_{c}}(k)
\end{array}\right] \\
& \quad \in \mathbb{R}^{l_{u} \times n_{c}\left(l_{u}+l_{y}\right)}
\end{aligned}
$$

is the controller gain matrix, and the regressor vector $\phi(k)$ is given by

$$
\phi(k) \triangleq\left[\begin{array}{c}
y(k-1) \\
\vdots \\
y\left(k-n_{c}\right) \\
u(k-1) \\
\vdots \\
u\left(k-n_{c}\right)
\end{array}\right] \in \mathbb{R}^{n_{c}\left(l_{u}+l_{y}\right)}
$$

We define the extended control vector $U(k) \in \mathbb{R}^{p_{c} l_{u}}$ by

$$
U(k) \triangleq\left[\begin{array}{c}
u(k-1) \\
\vdots \\
u\left(k-p_{c}\right)
\end{array}\right]
$$

where $p_{c} \geq q_{c}$. Note that if $p_{c}=q_{c}$, then $U(k)=U_{1}(k)$. From Eq. (11), it follows that the extended control vector $U(k)$ can be written as

$$
U(k)=\sum_{i=1}^{p_{c}} L_{i} \theta(k-i) \phi(k-i)
$$

where

$$
L_{i} \triangleq\left[\begin{array}{c}
0_{(i-1) l_{u} \times l_{u}} \\
I_{l_{u}} \\
0_{\left(p_{c}-i\right) l_{u} \times l_{u}}
\end{array}\right] \in \mathbb{R}^{p_{c} l_{u} \times l_{u}}
$$

Next, we define the retrospective performance vector $\hat{Z}(\hat{\theta}, k) \in \mathbb{R}^{p l_{z}}$ by

$$
\hat{Z}(\hat{\theta}, k) W_{z w} \phi_{z w}(k)+B_{z u} U_{1}(k)-\bar{B}_{z u}[U(k)-\hat{U}(\hat{\theta}, k)]
$$

where $\hat{\theta} \in \mathbb{R}^{l_{u} \times n_{c}\left(l_{u}+l_{y}\right)}$ is the surrogate controller gain matrix, $\bar{B}_{z u} \in$ $\mathbb{R}^{p l_{z} \times p_{c} l_{u}}$ is the surrogate input matrix, and

$$
\hat{U}(\hat{\theta}, k) \triangleq \sum_{i=1}^{p_{c}} L_{i} \hat{\theta} \phi(k-i)
$$

is the recomputed extended control vector. Substituting Eq. (6) into Eq. (17) yields

$$
\hat{Z}(\hat{\theta}, k)=Z(k)-\bar{B}_{z u}[U(k)-\hat{U}(\hat{\theta}, k)]
$$

Note that the expression for $\hat{Z}(\hat{\theta}, k)$ given by Eq. (19) does not depend on either the exogenous signal $w$ or the matrix $W_{z w}$, which includes information about the open-loop poles as well as the transfer function from $w$ to $z$. Hence, we do not need to know this model data, and when $w$ represents a disturbance, we do not need to assume that $w$ is known. However, when $w$ represents a command, then $w$ can be viewed as an additional measurement $y$, and thus the controller has feedforward action. The matrix $\bar{B}_{z u}$ is discussed in Sec. VI.

Note that Eq. (19) can be rewritten as

$$
\hat{Z}(\hat{\theta}, k)=f(k)+D(k) \operatorname{vec} \hat{\theta}
$$

where

$$
\begin{gathered}
f(k) \triangleq Z(k)-\bar{B}_{z u} U(k) \in \mathbb{R}^{p l_{z}} \\
D(k) \triangleq \sum_{i=1}^{p_{c}} \phi^{T}(k-i) \otimes\left(\bar{B}_{z u} L_{i}\right) \in \mathbb{R}^{p l_{z} \times n_{c} l_{u}\left(l_{u}+l_{y}\right)}
\end{gathered}
$$

vec is the column-stacking operator, and $\otimes$ represents the Kronecker product. Now consider the retrospective cost function

$$
\begin{aligned}
& J(\hat{\theta}, k) \triangleq \hat{Z}^{T}(\hat{\theta}, k) R_{1}(k) \hat{Z}(\hat{\theta}, k)+2 \hat{Z}^{T}(\hat{\theta}, k) R_{12}(k) \hat{u}(\hat{\theta}, k+1) \\
& \quad+\hat{u}^{T}(\hat{\theta}, k+1) R_{2}(k) \hat{u}(\hat{\theta}, k+1) \\
& \quad+\operatorname{tr}\left[R_{3}(k)(\hat{\theta}-\theta(k))^{T} R_{4}(k)(\hat{\theta}-\theta(k))\right]
\end{aligned}
$$

where $R_{1}(k) \in \mathbb{R}^{p l_{z} \times p l_{z}}, R_{12}(k) \in \mathbb{R}^{p l_{z} \times l_{u}}, R_{2}(k) \in \mathbb{R}^{l_{u} \times l_{u}}, R_{3}(k) \in$ $\mathbb{R}^{n_{c}\left(l_{u}+l_{y}\right) \times n_{c}\left(l_{u}+l_{y}\right)}, R_{4}(k) \in \mathbb{R}^{l_{u} \times l_{u}}$

$$
\left[\begin{array}{cc}
R_{1}(k) & R_{12}(k) \\
R_{12}^{T}(k) & R_{2}(k)
\end{array}\right]
$$

is positive semidefinite, $R_{3}(k)$ and $R_{4}(k)$ are positive definite, and

$$
\hat{u}(\hat{\theta}, k) \triangleq \hat{\theta} \phi(k)
$$

Substituting Eq. (20) into Eq. (23) yields

$$
J(\hat{\theta}, k)=(\operatorname{vec} \hat{\theta})^{T} M(k) \operatorname{vec} \hat{\theta}+b^{T}(k) \operatorname{vec} \hat{\theta}+c(k)
$$

where

$$
\begin{aligned}
& M(k) \triangleq D^{T}(k) R_{1}(k) D(k)+2 D^{T}(k)\left[\phi^{T}(k) \otimes R_{12}(k)\right] \\
& \quad+\left[\phi(k) \phi^{T}(k)\right] \otimes R_{2}(k)+R_{3}(k) \otimes R_{4}(k) \\
& b(k) \triangleq 2 D^{T}(k) R_{1}(k) f(k)+2\left[\phi(k) \otimes R_{12}^{T}(k)\right] f(k) \\
& \quad-2\left[R_{3}(k) \otimes R_{4}(k)\right] \operatorname{vec} \theta(k)
\end{aligned}
$$

$$
c(k) \triangleq f^{T}(k) R_{1}(k) f(k)+\operatorname{tr}\left[R_{3}(k) \theta^{T}(k) R_{4}(k) \theta(k)\right]
$$

Since $M(k)$ is positive definite, $J(\hat{\theta}, k)$ has the strict global minimizer $\theta(k+1)$ given by

$$
\theta(k+1)=-\frac{1}{2} \operatorname{vec}^{-1}\left[M^{-1}(k) b(k)\right]
$$

Equation (29) is the adaptive control update law. Note that $\bar{B}_{z u}$ (which appears in $f(k)$ and $D(k))$ must be specified in order to implement Eq. (29). Furthermore, Eq. (29) requires the online inversion of a positive-definite matrix of size $n_{c} l_{u}\left(l_{u}+l_{y}\right) \times n_{c} l_{u}\left(l_{u}+l_{y}\right)$.

In the special case

$$
\begin{gathered}
R_{1}(k) \triangleq I_{p l_{z}}, \quad R_{12}(k) \triangleq 0_{p l_{z} \times l_{u}}, \quad R_{2}(k) \triangleq 0_{l_{u} \times l_{u}} \\
R_{3}(k) \triangleq \alpha(k) I_{n_{c}\left(l_{u}+l_{y}\right)}, \quad R_{4}(k) \triangleq I_{l_{u}}
\end{gathered}
$$

where $\alpha(k)>0$ is a scalar, Eqs. (26-28) become

$$
\begin{gathered}
M(k)=D^{T}(k) D(k)+\alpha(k) I_{n_{c} l_{u}\left(l_{u}+l_{y}\right)} \\
b(k)=2 D^{T}(k) f(k)-2 \alpha(k) \operatorname{vec} \theta(k) \\
c(k)=f^{T}(k) f(k)+\alpha(k) \operatorname{tr}\left[\theta^{T}(k) \theta(k)\right]
\end{gathered}
$$

Using the matrix inversion lemma, it follows that

$$
\begin{aligned}
& M^{-1}(k)=\alpha^{-1}(k) I_{n_{c} l_{u}\left(l_{u}+l_{y}\right)} \\
& \quad-\alpha^{-1}(k) D^{T}(k)\left[\alpha(k) I_{p l_{z}}+D(k) D^{T}(k)\right]^{-1} D(k)
\end{aligned}
$$

Consequently, in this case, the update law (29) requires the online inversion of a positive-definite matrix of size $p l_{z} \times p l_{z}$. We use the weightings (30) and (31) for all of the examples in this paper. The weighting parameter $\alpha \overline{(k)}$ introduced in Eq. (31) is called the learning rate, since it affects the convergence speed of the adaptive control algorithm. As $\alpha(k)$ is increased, a higher weight is placed on the difference between the previous controller coefficients and the updated controller coefficients and, as a result, convergence speed is 


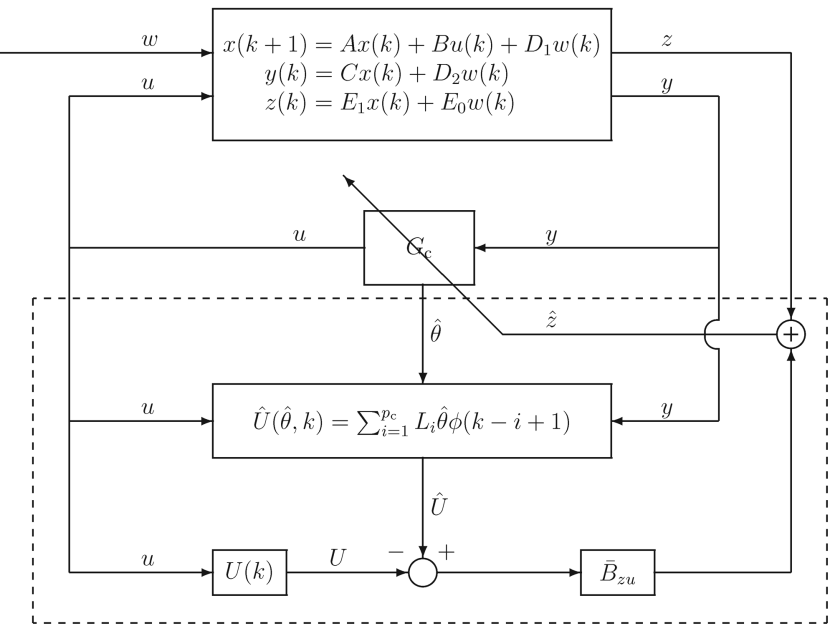

Fig. 1 Closed-loop system including adaptive control algorithm with the retrospective correction filter (dashed box) for $p=1$.

lowered. Likewise, as $\alpha(k)$ is decreased, convergence speed is raised. By varying $\alpha(k)$, we can effect tradeoffs between transient performance and convergence speed.

We define the retrospective performance variable $\hat{z} \in \mathbb{R}^{l_{z}}$ by

$$
\hat{z}(k) \triangleq\left[\begin{array}{llll}
I_{l_{z}} & 0_{l_{z} \times l_{z}} & \cdots & 0_{l_{z} \times l_{z}}
\end{array}\right] \hat{Z}(\theta(k), k)
$$

In the particular case of $z=y$, using $\hat{z}$ in place of $y$ in the regressor vector (13) yields faster convergence. Therefore, for $z=y$, we redefine $\overline{\mathrm{Eq}}$. (13) as

$$
\phi(k) \triangleq\left[\begin{array}{c}
\hat{z}(k-1) \\
\vdots \\
\hat{z}\left(k-n_{c}\right) \\
u(k-1) \\
\vdots \\
u\left(k-n_{c}\right)
\end{array}\right]
$$

The novel feature of the adaptive control algorithm given by Eqs. (11) and (29) is the use of the RCF (19), as shown in Fig. 1 for $p=1$. RCF provides an inner loop to the adaptive control law by modifying the extended performance vector $Z(k)$ in terms of the difference between the actual past control inputs $U(k)$ and the recomputed control inputs $\hat{U}(\hat{\theta}, k)$.

\section{Markov-Parameter Polynomial}

By recursively substituting Eq. (1) into Eq. (3), it follows that $z(k)$ can be represented by

$$
\begin{aligned}
& z(k)=E_{1} A^{r} x(k-r)+H_{1} u(k-1)+H_{2} u(k-2)+\cdots \\
& +H_{r} u(k-r)+H_{z w, 0} w(k)+H_{z w, 1} w(k-1)+\cdots \\
& +H_{z w, r} w(k-r)
\end{aligned}
$$

where $r \geq d$ and $H_{z w, 0} \triangleq E_{0}$, and $H_{z w, i} \triangleq E_{1} A^{i-1} D_{1}$ for all $i>0$. In terms of the backward-shift operator $\mathbf{q}^{-1}$, Eq. (38) can be rewritten as

$$
\begin{aligned}
& z(k)=E_{1} A^{r} \mathbf{q}^{-r} x(k)+\left[H_{1} \mathbf{q}^{-1}+H_{2} \mathbf{q}^{-2}+\cdots+H_{r} \mathbf{q}^{-r}\right] u(k) \\
& +\left[H_{z w, 0}+H_{z w, 1} \mathbf{q}^{-1}+\cdots+H_{z w, r} \mathbf{q}^{-r}\right] w(k)
\end{aligned}
$$

Shifting Eq. (39) forward by $r$ steps gives

$$
z(k+r)=E_{1} A^{r} x(k)+p_{r}(\mathbf{q}) u(k)+W_{r}(\mathbf{q}) w(k)
$$

where $\mathbf{q}$ is the forward-shift operator,

$$
W_{r}(\mathbf{q}) \triangleq H_{z w, 0} \mathbf{q}^{r}+H_{z w, 1} \mathbf{q}^{r-1}+H_{z w, 2} \mathbf{q}^{r-2}+\cdots+H_{z w, r}
$$

and

$$
p_{r}(\mathbf{q}) \triangleq H_{1} \mathbf{q}^{r-1}+H_{2} \mathbf{q}^{r-2}+\cdots+H_{r}
$$

We call $p_{r}(\mathbf{q})$ the Markov-parameter polynomial. Note that $p_{r}(\mathbf{q})$ is a matrix polynomial in the MIMO case and a polynomial in the SISO case. Furthermore, since $H_{1}=\cdots=H_{d-1}=0$ when $d \geq 2$, it follows that $p_{r}(\mathbf{q})$ for all $r \geq d \geq 1$ can be written as

$$
p_{r}(\mathbf{q})=H_{d} \mathbf{q}^{r-d}+H_{d+1} \mathbf{q}^{r-d-1}+\cdots+H_{r}
$$

The Markov-parameter polynomial $p_{r}(\mathbf{q})$ contains information about the relative degree $d$ and, in the SISO case, the sign of the highfrequency gain: that is, the sign of $H_{d}$. We show below that $p_{r}(\mathbf{q})$ also contains information about the transmission zeros of $G_{z u}(\mathbf{z}) \triangleq$ $E_{1}(\mathbf{z} I-A)^{-1} B$, which is given by

$$
G_{z u}(\mathbf{z})=\frac{1}{\mathbf{z}^{n}+\alpha_{1} \mathbf{z}^{n-1}+\cdots+\alpha_{n}}\left(\beta_{1} \mathbf{z}^{n-1}+\beta_{2} \mathbf{z}^{n-2}+\cdots+\beta_{n}\right)
$$

To relate the transmission zeros of $G_{z u}$ to $p_{r}(\mathbf{q})$, the Laurent series expansion of $G_{z u}$ about $\mathbf{z}=\infty$ is given by

$$
G_{z u}(\mathbf{z})=\sum_{i=1}^{\infty} \mathbf{z}^{-i} H_{i}
$$

This expansion converges uniformly on all compact subsets of $\{\mathbf{z}:|\mathbf{z}|>\rho(A)\}$, where $\rho(A)$ is the spectral radius of $A$ ([2] ], Theorem 13, page 186). By truncating the summation in Eq. (45), we obtain the truncated Laurent expansion $\bar{G}_{r, z u}$ of $G_{z u}$, given by

$$
\begin{aligned}
& \bar{G}_{r, z u}(\mathbf{z}) \triangleq \sum_{i=1}^{r} \mathbf{z}^{-i} H_{i}=\frac{1}{\mathbf{z}^{r}}\left(H_{1} \mathbf{z}^{r-1}+\cdots+H_{r-1} \mathbf{z}+H_{r}\right) \\
& \quad=\frac{1}{\mathbf{z}^{r}} p_{r}(\mathbf{z})
\end{aligned}
$$

Consequently, the Markov-parameter polynomial $p_{r}(\mathbf{q})$ is closely related to the truncated Laurent expansion of $G_{z u}$.

\section{A. Approximation of Outer Non-Minimum-Phase Zeros}

In the case of MIMO systems, $p_{r}(\mathbf{q})$ is a matrix polynomial and thus does not have roots in the sense of a polynomial. We therefore require the notion of a Smith zero ([29], page 259). Specifically, $\mathbf{z} \in \mathbb{C}$ is a Smith zero of $p_{r}(\mathbf{q})$ if the rank of $p_{r}(\mathbf{z})$ is less than the normal rank of $p_{r}(\mathbf{q})$ : that is, the maximum rank of $p_{r}(\xi)$ taken over all $\xi \in \mathbb{C}$.

Let $\xi \in \mathbb{C}$ be a transmission zero of $G_{z u}$. Then $\xi$ is an outer zero of $G_{z u}$ if $|\xi| \geq \rho(A)$. Otherwise, $\xi$ is an inner zero of $G_{z u}$.

The following result shows that the Smith zeros of the Markovparameter polynomial $p_{r}(\mathbf{q})$ asymptotically approximate each outer transmission zero of $G_{z u}$.

Fact 1. Let $\xi \in \mathbb{C}$ be an outer transmission zero of $G_{z u}$. For each $r$, let $\mathcal{R}_{r} \triangleq\left\{\xi_{r, 1}, \ldots, \xi_{r, m_{r}}\right\}$ denote the set of Smith zeros of $p_{r}(\mathbf{q})$. Then there exists a sequence $\left\{\xi_{r, i_{r}}\right\}_{r=1}^{\infty}$ that converges to $\xi$ as $r \rightarrow \infty$.

The following specialization to SISO transfer functions shows that the roots of $p_{r}(\mathbf{q})$ asymptotically approximate each outer zero of $G_{z u}$.

Fact 2. Consider $l_{u}=l_{z}=1$, and let $\xi \in \mathbb{C}$ be an outer zero of $G_{z u}$ For each $r$, let $\mathcal{R}_{r} \triangleq\left\{\xi_{r, 1}, \ldots, \xi_{r, r-d}\right\}$ be the set of roots of $p_{r}(\mathbf{q})$. Then there exists a sequence $\left\{\xi_{r, i_{r}}\right\}_{r=1}^{\infty}$ that converges to $\xi$ as $r \rightarrow \infty$.

The following examples illustrate Fact 2 by showing that as $r$ increases, roots of the Markov-parameter polynomial $p_{r}(\mathbf{q})$, and hence roots of the numerator of the truncated transfer function $\bar{G}_{r, z u}$, asymptotically approximate each outer non-minimum-phase zero of $G_{z u}$. The remaining roots of $p_{r}(\mathbf{q})$ are either located at the origin or form an approximate ring with radius close to $\rho(A)$. These roots are spurious and have no effect on the adaptive control algorithm.

Example 1 (SISO, non-minimum-phase, stable plant). Consider the plant $G_{z u}$ with $d=2 ; H_{2}=1$; poles $0.5 \pm 0.5 \jmath,-0.5 \pm 0.5 \mathrm{~J}$, \pm 0.95 , and $\pm 0.7 \jmath$; minimum-phase zeros $0.3 \pm 0.7 \jmath$ and $-0.7 \pm 0.3 \mathrm{~J}$; and outer non-minimum-phase zeros 1.25 and -1.5 . Table 1 lists the approximated non-minimum-phase zeros obtained as roots of $p_{r}(\mathbf{q})$ as a function of $r$. Note that as $r$ increases, the outer non-minimum-phase zeros are more closely approximated by the roots of $p_{r}(\mathbf{q})$ (see Fig. 2). 
Table 1 Approximated non-minimum-phase zeros obtained as roots of $p_{r}(q)$ as a function of $r$ for the stable non-minimum-phase plant in Example 1

\begin{tabular}{ll}
\hline \hline$r^{\mathrm{a}}$ & $\operatorname{roots}_{\mathrm{nmp}}\left(p_{r}(\mathbf{q})\right)$ \\
\hline 6 & $\{0.944,-1.537\}$ \\
8 & $\{1.170,-1.502\}$ \\
10 & $\{1.207,-1.498\}$ \\
15 & $\{1.240,-1.499\}$ \\
20 & $\{1.248,-1.500\}$ \\
25 & $(1.250,-1.500\}$ \\
\hline \hline & ${ }^{\mathrm{a} A s} r$ increases, the outer zeros are more accurately modeled.
\end{tabular}

Example 2 (SISO, non-minimum-phase, unstable plant). Consider the plant $G_{z u}$ with $d=2 ; H_{2}=1$; poles $0.5 \pm 0.5 \mathrm{~J},-0.5 \pm 0.5 \mathrm{~J}$, $\pm 0.7 \mathrm{~J},-0.95$, and 1.4 ; minimum-phase zeros $0.3 \pm 0.7 \mathrm{~J}$ and $-0.7 \pm 0.3 \mathrm{j}$; outer non-minimum-phase zero -1.5 ; and inner nonminimum-phase zero 1.25 . Figure 3 shows the roots of $p_{25}(\mathbf{q})$. Note that the root of $p_{25}(\mathbf{q})$ outside $\bar{\rho}(A)$ is close to the outer nonminimum-phase zero -1.5 . However, the inner non-minimum-phase zero 1.25 is not approximated by a root of $p_{25}(\mathbf{q})$. The remaining roots are either located at the origin or form an approximate ring with radius close to $\rho(A)$.

\section{B. Approximation of Inner Non-Minimum-Phase Zeros}

Example 2 illustrates that the roots of $p_{r}(\mathbf{q})$ approximate each outer non-minimum-phase zero of $G_{z u}$. However, inner nonminimum-phase zeros of $G_{z u}$ are not approximated by roots of $p_{r}(\mathbf{q})$. To overcome this deficiency, we can use information about the plant's unstable poles to create a modified Markov-parameter polynomial $\tilde{p}_{r}(\mathbf{q})$ whose roots approximate each non-minimumphase zero of $G_{z u}$. For illustration, assume that the SISO plant $G_{z u}$ has a unique unstable pole $\xi \in \mathbb{C}$ whose absolute value is greater than all other poles of $G_{z u}$. Then we define

$$
\begin{aligned}
\tilde{G}_{z u}(\mathbf{z}) & \triangleq \frac{\mathbf{z}-\zeta}{\mathbf{z}} G_{z u}(\mathbf{z}) \\
= & G_{z u}(\mathbf{z})-\frac{\zeta}{\mathbf{z}} G_{z u}(\mathbf{z}) \\
& =\sum_{i=d}^{\infty} \mathbf{z}^{-i} H_{i}-\sum_{i=d}^{\infty} \mathbf{z}^{-(i+1)} \zeta H_{i} \\
& =\sum_{i=d}^{\infty} \mathbf{z}^{-i}\left[H_{i}-\zeta H_{i-1}\right] \\
& =\sum_{i=d}^{\infty} \mathbf{z}^{-i} \tilde{H}_{i}
\end{aligned}
$$

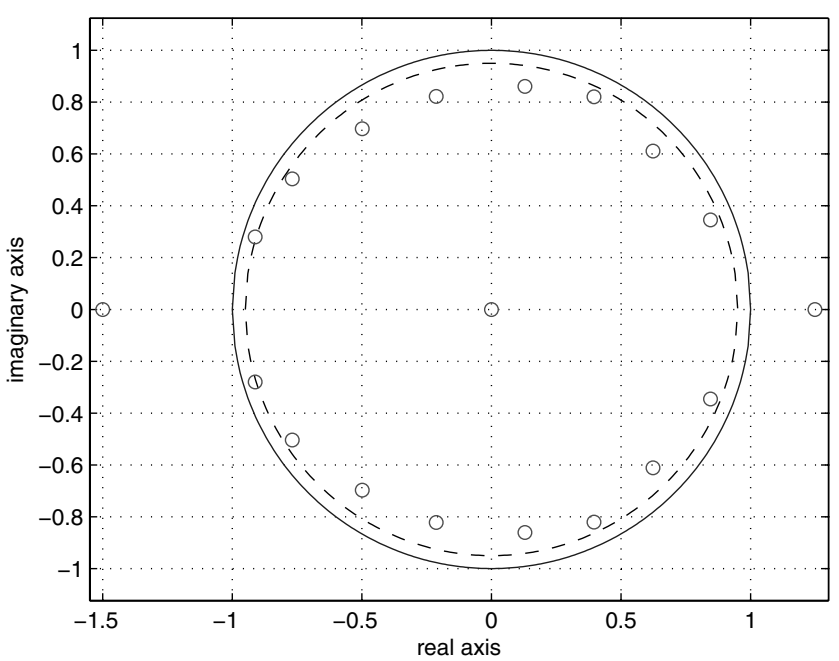

Fig. 2 Roots of $p_{20}(q)$ for the stable, non-minimum-phase plant in Example 1. The dashed line denotes $\rho(A)=0.95$. Note that the roots outside $\rho(A)$ are close to the outer non-minimum-phase zeros -1.5 and 1.25. The remaining roots are either located at the origin or form an approximate ring with radius close to $\rho(A)$.

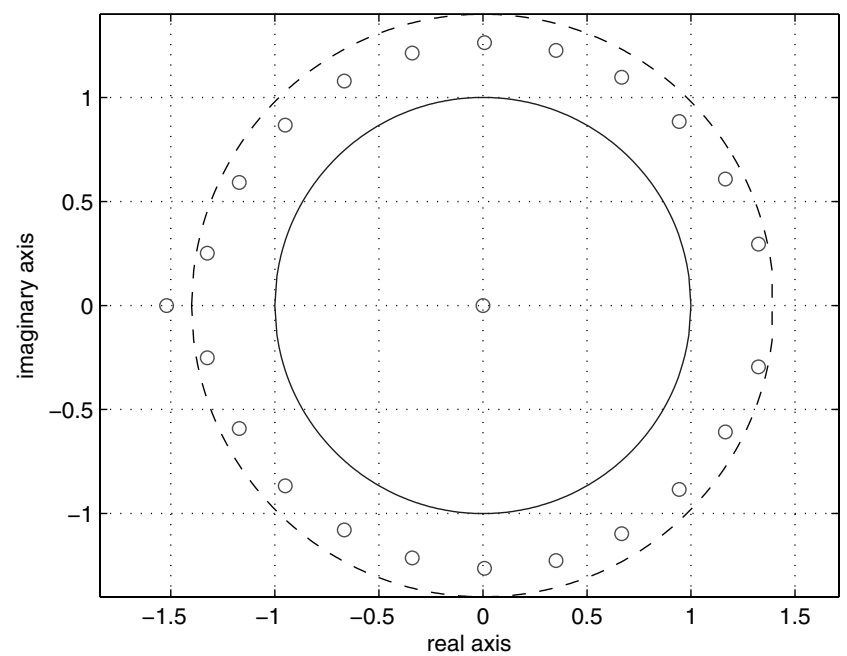

Fig. 3 Roots of $p_{25}(q)$ for the unstable, non-minimum-phase plant in Example 2. The dashed line denotes $\rho(A)=1.4$. Note that the root of $p_{25}(q)$ outside $\rho(A)$ is close to the outer non-minimum-phase zero -1.5 . However, the non-minimum-phase zero 1.25 is not approximated by a root of $p_{25}(q)$. The remaining roots are either located at the origin or form an approximate ring with radius close to $\rho(A)$.

where $\tilde{H}_{i} \triangleq H_{i}-\zeta H_{i-1}$ are the modified Markov parameters for $i=1,2, \ldots$, and $H_{0}=0$. By repeating this operation for each unstable pole of $G_{z u}$, the roots of the modified Markov-parameter polynomial

$$
\tilde{p}_{r}(\mathbf{q}) \triangleq \tilde{H}_{d} \mathbf{q}^{r-d}+\tilde{H}_{d+1} \mathbf{q}^{r-d-1}+\cdots+\tilde{H}_{r}
$$

can approximate each non-minimum-phase zero of $G_{z u}$. The following example illustrates this process.

Example 3 (Example 2 with pole information). Reconsider Example 2, where the inner non-minimum-phase zero 1.25 is not approximated by a root of $p_{r}(\mathbf{q})$. Using knowledge of the unstable pole 1.4 to construct $\tilde{p}_{r}(\mathbf{q})$ given by Eq. (48), Fig. 4 shows the roots of $\tilde{p}_{25}(\mathbf{q})$. Note that the roots outside $\rho(\tilde{A})$, where $\tilde{A}$ is the dynamics matrix of a minimal realization of $\tilde{G}_{z u}$, are close to the nonminimum-phase zeros of $G_{z u}$. The remaining roots are either located at the origin or form an approximate ring with radius close to $\rho(\tilde{A})$.

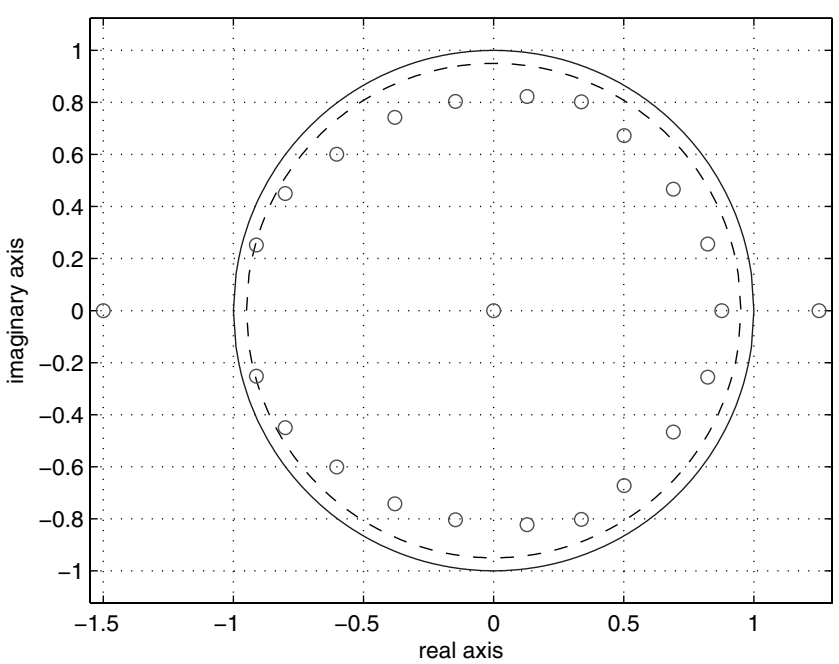

Fig. 4 Roots of $\tilde{p}_{25}(q)$ for the unstable, non-minimum-phase plant in Example 3. The dashed line denotes $\rho(\tilde{A})=0.95$, where $\tilde{A}$ is the dynamics matrix of a minimal realization of $\tilde{G}_{z u}$. Note that the roots outside $\rho(\tilde{A})$ are close to the inner and outer non-minimum-phase zeros of $G_{z u}$. The remaining roots are either located at the origin or form an approximate ring with radius close to $\rho(\tilde{A})$. 


$$
\begin{array}{ccc}
\alpha_{1, i} \triangleq-\alpha_{i}, & \beta_{1, i} \triangleq \beta_{i}, & \gamma_{1, i} \triangleq \gamma_{i}, \\
\vdots & \vdots & \vdots \\
\alpha_{r, i} \triangleq \alpha_{r-1,1} \alpha_{1, i}+\alpha_{r-1, i+1}, & \beta_{r, i} \triangleq \alpha_{r-1,1} \beta_{1, i}+\beta_{r-1, i+1}, & \gamma_{r, i} \triangleq \alpha_{r-1,1} \gamma_{1, i}+\gamma_{r-1, i+1}, \\
\vdots & \vdots & \vdots \\
\alpha_{r, n} \triangleq \alpha_{r-1,1} \alpha_{1, n}, & \beta_{r, n} \triangleq \alpha_{r-1,1} \beta_{1, n}, & \gamma_{r, n} \triangleq \alpha_{r-1,1} \gamma_{1, n}
\end{array}
$$

\section{Construction of $\bar{B}_{z u}$}

We present four constructions for $\bar{B}_{z u}$ based on the available modeling information.

\section{A. $B_{z u}$-Based Construction}

If $B_{z u}$ given by Eq. (8) is known, then $\bar{B}_{z u}$ can be chosen to be equal to $B_{z u}$, with $p_{c}=q_{c}$. In this case, $U(k)=U_{1}(k)$, and Eq. (17) becomes

$$
\hat{Z}(\hat{\theta}, k)=W_{z w} \phi_{z w}(k)+B_{z u} \hat{U}(\hat{\theta}, k)
$$

This construction of $\bar{B}_{z u}$ captures information about the relative degree $d$, the first nonzero Markov parameter (since $H_{d}=\beta_{d}$ ), and exact values of all transmission zeros of $G_{z u}$ : that is, both minimum-phase and non-minimum-phase transmission zeros.

\section{B. Non-Minimum-Phase-Zero-Based Construction}

Consider $l_{u}=l_{z}=1$ and assume that $H_{d}$ and the non-minimumphase zeros of $G_{z u}$ are known. Then we define the non-minimumphase-zero polynomial $N(\mathbf{q})$ to be the polynomial whose roots are equal to the non-minimum-phase zeros of $G_{z u}$ : that is,

$$
N(\mathbf{q}) \triangleq H_{d} \mathbf{q}^{m}+\tilde{\beta}_{1} \mathbf{q}^{m-1}+\cdots+\tilde{\beta}_{m}
$$

where $m \geq 0$ is the number of non-minimum-phase zeros in $G_{z u}$, and $\tilde{\beta}_{1}, \ldots, \tilde{\beta}_{m} \in \mathbb{R}$. If $m=0$, that is, $G_{z u}$ is minimum phase, then $N(\mathbf{q})=H_{d}$. With $p_{c}=q_{c}$, the non-minimum-phase-zero-based construction of $\bar{B}_{z u}$ is thus given by
Note that $\beta_{r, 1}=H_{r}$ and $\gamma_{r, 1}=H_{z w, r}$. We represent Eq. (52) with $w=0$ as the $r$-Markov transfer function:

$$
\begin{aligned}
& G_{r, z u}(\mathbf{z})=\frac{1}{\mathbf{z}^{r+n-1}+\alpha_{r, 1} \mathbf{z}^{n-1}+\cdots+\alpha_{r, n}} \cdot\left(H_{1} \mathbf{z}^{r+n-2}+\cdots\right. \\
& \left.\quad+H_{r-1} \mathbf{z}^{n}+H_{r} \mathbf{z}^{n-1}+\beta_{r, 2} \mathbf{z}^{n-2}+\cdots+\beta_{r, n}\right)
\end{aligned}
$$

The system representation (54) is nonminimal, since its order is $n+r-1$, and thus Eq. (54) includes poles that are not present in the original model. Furthermore, note that the coefficients of the terms $\mathbf{z}^{n+r-2}$ through $\mathbf{z}^{n}$ in the denominator are zero. These facts are irrelevant for the following development. Using the numerator coefficients of Eq. (54), the $r$-Markov-based construction of $\bar{B}_{z u}$ with $p_{c}=q_{c}+r-1$ is given by

$$
\begin{aligned}
& \bar{B}_{z u} \\
& =\left[\begin{array}{ccccccccc}
H_{1} & \cdots & H_{r} & \beta_{r, 2} & \cdots & \beta_{r, n} & 0_{l_{z} \times l_{u}} & \cdots & 0_{l_{z} \times l_{u}} \\
0_{l_{z} \times l_{u}} & \ddots & & \ddots & \ddots & & \ddots & \ddots & \vdots \\
\vdots & \ddots & \ddots & & \ddots & \ddots & & \ddots & 0_{l_{z} \times l_{u}} \\
0_{l_{z} \times l_{u}} & \cdots & 0_{l_{z} \times l_{u}} & H_{1} & \cdots & H_{r} & \beta_{r, 2} & \cdots & \beta_{r, n}
\end{array}\right]
\end{aligned}
$$

This construction of $\bar{B}_{z u}$ captures information about the relative degree $d$, the first nonzero Markov parameter, and exact values of all

$$
\bar{B}_{z u}=\left[\begin{array}{cccccccccccc}
H_{1} & \cdots & H_{d} & \tilde{\beta}_{1} & \cdots & \tilde{\beta}_{m} & 0_{l_{z} \times l_{u}} & \cdots & 0_{l_{z} \times l_{u}} & 0_{l_{2} \times l_{u}} & \cdots & 0_{l_{z} \times l_{u}} \\
0_{l_{z} \times l_{u}} & \ddots & & \ddots & \ddots & & \ddots & \ddots & & \ddots & \ddots & \vdots \\
\vdots & \ddots & \ddots & & \ddots & \ddots & & \ddots & \ddots & & \ddots & \vdots \\
0_{l_{z} \times l_{u}} & \cdots & 0_{l_{z} \times l_{u}} & H_{1} & \cdots & H_{d} & \tilde{\beta}_{1} & \cdots & \tilde{\beta}_{m} & 0_{l_{z} \times l_{u}} & \cdots & 0_{l_{z} \times l_{u}}
\end{array}\right]
$$

where $H_{1}=\cdots=H_{d-1}=0$. This construction of $\bar{B}_{z u}$ captures information about the relative degree $d$, the first nonzero Markov parameter, and exact values of all non-minimum-phase zeros of $G_{z u}$. In the minimum-phase case, the only required modeling information is $H_{d}$. This construction of $\bar{B}_{z u}$ can be extended to the MIMO case by replacing each minimum-phase zero in the Smith-McMillan form of $G_{z u}$ by a zero at $\mathbf{z}=0$; for details, see [26].

\section{C. $r$-Markov-Based Construction}

Replacing $k$ with $k-1$ in Eq. (4) and substituting the resulting relation back into Eq. (4) yields a 2 -Markov model. Repeating this procedure $r-1$ times yields the $r$-Markov model of Eqs. (1- $\underline{3})$ :

$$
\begin{aligned}
z(k) & =\sum_{i=1}^{n} \alpha_{r, i} z(k-r-i+1)+\sum_{i=d}^{r-1} H_{i} u(k-i) \\
& +\sum_{i=1}^{n} \beta_{r, i} u(k-r-i+1)+\sum_{i=0}^{r-1} H_{z w, i} w(k-i) \\
& +\sum_{i=1}^{n} \gamma_{r, i} w(k-r-i+1)
\end{aligned}
$$

where, for $i=1, \ldots, n$, the coefficients $\alpha_{r, i} \in \mathbb{R}, \beta_{r, i} \in \mathbb{R}^{l_{2} \times l_{u}}$, and $\gamma_{r, i} \in \mathbb{R}^{l_{z} \times l_{w}}$ are given by transmission zeros of $G_{z u}$ : that is, both minimum-phase and nonminimum-phase transmission zeros.

\section{Markov-Parameter-Based Construction}

Using the numerator coefficients of Eq. (46), the Markovparameter-based construction of $\bar{B}_{z u}$ with $p_{c}=\bar{q}_{c}+r-1$ is given by

$\bar{B}_{z u}=\left[\begin{array}{ccccccccc}H_{1} & \cdots & H_{r} & 0_{l_{z} \times l_{u}} & \cdots & 0_{l_{z} \times l_{u}} & 0_{l_{z} \times l_{u}} & \cdots & 0_{l_{z} \times l_{u}} \\ 0_{l_{z} \times l_{u}} & \ddots & & \ddots & \ddots & & \ddots & \ddots & \vdots \\ \vdots & \ddots & \ddots & & \ddots & \ddots & & \ddots & \vdots \\ 0_{l_{z} \times l_{u}} & \cdots & 0_{l_{z} \times l_{u}} & H_{1} & \cdots & H_{r} & 0_{l_{z} \times l_{u}} & \cdots & 0_{l_{z} \times l_{u}}\end{array}\right]$

The Markov parameters are the numerator coefficients of a truncated Laurent series expansion of $G_{z u}$ about $\mathbf{z}=\infty$. The Markov parameters contain information about the relative degree $d$ and, as shown by Fact 2 for the SISO case, approximate values of all outer nonminimum-phase zeros of $G_{z u}$. The advantage in using $\bar{B}_{z u}$ given by Eq. (56) rather than Eq. (55) is that $\beta_{r, 2}, \ldots, \beta_{r, n}$ need not be known. If, however, $G_{z u}$ has inner non-minimum-phase zeros and the 
unstable poles of $G_{z u}$ whose absolute values are greater than at least one inner non-minimum-phase zero are known, then we can replace the Markov parameters $H_{1}, \ldots, H_{r}$ in Eq. (56) by the modified Markov parameters $\tilde{H}_{1}, \ldots, \tilde{H}_{r}$ given in Eq. (47). If these poles are not known, then $\bar{B}_{z u}$ can be chosen to be either $B_{z u}$, the nonminimum-phase-zero form in Eq. (51), or the $r$-Markov form in Eq. (55).

Note that if the order $n$ of the system is known and $2 n+1$ Markov parameters are available, then a state-space model of the system can be reconstructed by using the eigensystem realization algorithm [30]. However, the examples considered in Secs. VII and VIII use substantially fewer Markov parameters.

\section{Numerical Examples: Nominal Cases}

We now present numerical examples to illustrate the response of the RCF adaptive control algorithm under nominal conditions. We consider a sequence of examples of increasing complexity, ranging from SISO minimum-phase plants to MIMO non-minimum-phase plants, including stable and unstable cases. Each SISO example is constructed such that $H_{d}=1$. All examples assume $y=z$, with $\phi(k)$ given by Eq. (37), and in all simulations, the adaptive controller gain matrix $\theta(\bar{k})$ is initialized to zero. Unless otherwise noted, all examples assume $x(0)=0$.

Example 4 (SISO, minimum-phase, unstable plant, stabilization). Consider the plant $G_{z u}$ with $d=1$, poles 0 and 1.5, and inner nonminimum-phase zero -1.25 . For stabilization, we take $D_{1}$ and $E_{0}$ to be zero matrices. Let $\bar{B}_{z u}$ be given by Eq. ( $\left.\underline{51}\right)$, which is constructed using the first nonzero Markov parameter $\overline{H_{1}}=1$ and the location of the non-minimum-phase zero -1.25 : that is, $N(\mathbf{q})=\mathbf{q}+1.25$. We take $n_{c}=2, p=1$, and $\alpha(k) \equiv 10$. The closed-loop response is shown in Fig. 5 for $x(0)=\left[\begin{array}{ll}0.1 & 0.4\end{array}\right]^{T}$.

Example 5 (SISO, minimum-phase, unstable plant, commandfollowing). Consider the double integrator plant $G_{z u}$ with $d=3$; poles $0.5 \pm 0.5 \jmath,-0.5 \pm 0.5 \jmath, 1$, and 1 ; and minimum-phase zeros $0.3 \pm 0.7 j$ and 0.5 . We consider a command-following problem with step command $w(k)=1$. With the plant realized in controllable canonical form, we take $D_{1}=0$ and $E_{0}=-1$. We take $n_{c}=10$, $p=5, \alpha(k) \equiv 5$, and $r=10$, with $\bar{B}_{z u}$ given by Eq. (56). The closedloop response is shown in Fig. $\underline{6}$.

Example 6 (SISO, minimum-phase, stable plant, commandfollowing, disturbance rejection). Consider the plant $G_{z u}$ with $d=3$; poles $0.5 \pm 0.5 \jmath,-0.5 \pm 0.5 \jmath, \pm 0.9$, and $\pm 0.7 \jmath$; and minimumphase zeros $0.3 \pm 0.7 \jmath,-0.7 \pm 0.3 \jmath$, and 0.5 . We consider a combined step-command-following and disturbance-rejection problem with command $w_{1}$ and disturbance $w_{2}$ given by
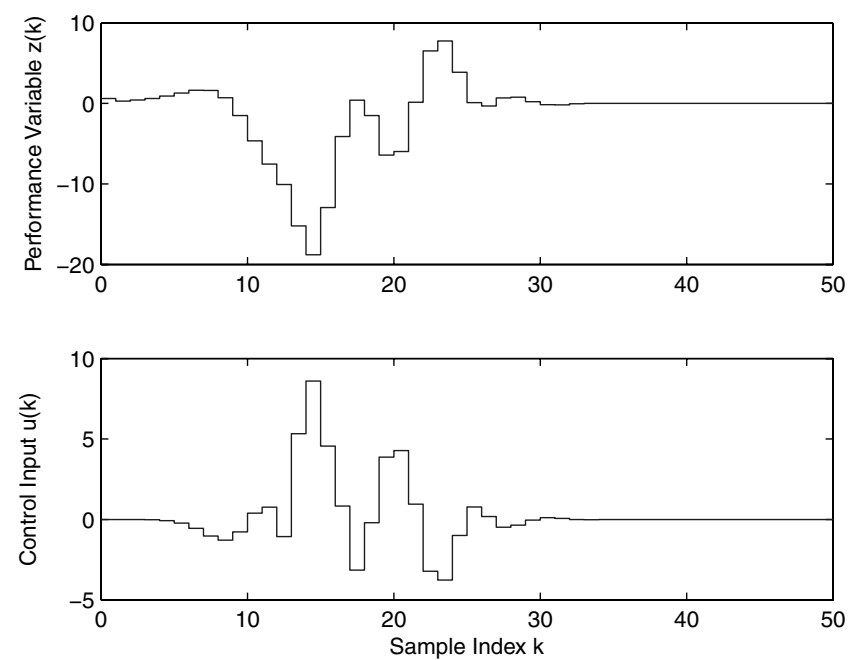

Fig. 5 Closed-loop response of the unstable, minimum-phase, SISO plant in Example 4 using the non-minimum-phase-zero-based construction Eq. (51) of $\bar{B}_{z u}$. The control is turned on at $k=0$. The controller order is $n_{c}=2$ with parameters $p=1$ and $\alpha(k) \equiv 10$.
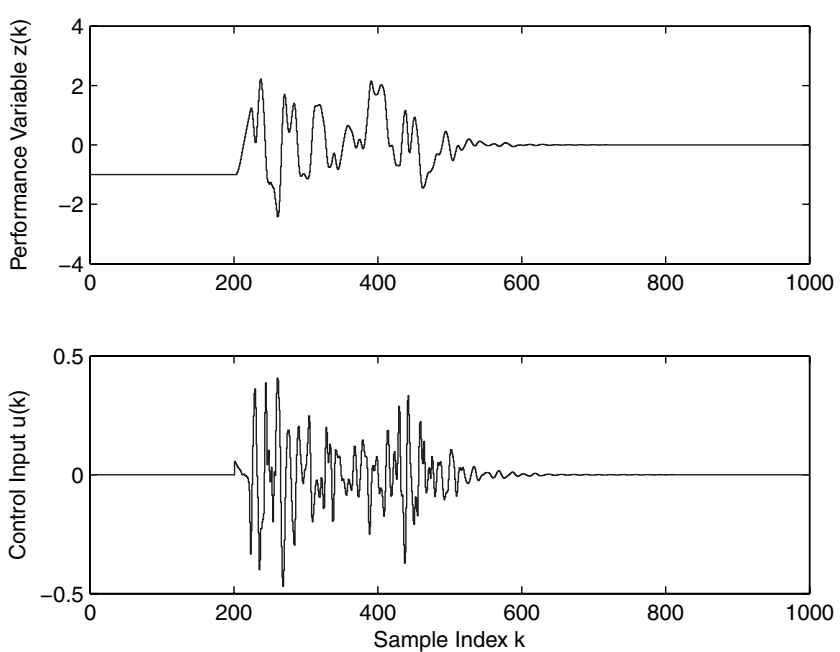

Fig. 6 Closed-loop response of the unstable, minimum-phase, SISO plant in Example 5 with a step command. The control is turned on at $k=200$. The controller order is $n_{c}=10$ with parameters $p=5$, $\alpha(k) \equiv 5$, and $r=10$, with $\bar{B}_{z u}$ given by Eq. ( $\underline{56}$.

$$
w(k)=\left[\begin{array}{c}
w_{1}(k) \\
w_{2}(k)
\end{array}\right]=\left[\begin{array}{c}
5 \\
\sin \Omega_{1} k
\end{array}\right]
$$

where $\Omega_{1}=\pi / 10 \mathrm{rad} /$ sample. With the plant realized in controllable canonical form, we take

$$
D_{1}=\left[\begin{array}{ll}
0 & 0 \\
0 & 1
\end{array}\right]
$$

and $E_{0}=\left[\begin{array}{ll}-1 & 0\end{array}\right]$. The disturbance, which is not matched, is assumed to be unknown, and the command signal is not used directly. We take $n_{c}=20, p=1, \alpha(k) \equiv 50$, and $r=3$, with $\bar{B}_{z u}$ given by Eq. (56). The closed-loop response is shown in Fig. 7.

The following examples are disturbance-rejection simulations, that is, $E_{0}=0$, with the unknown two-tone sinusoidal disturbance:

$$
w(k)=\left[\begin{array}{c}
\sin \Omega_{1} k \\
-1.5 \sin \Omega_{2} k
\end{array}\right]
$$

where $\Omega_{1}=\pi / 10 \mathrm{rad} /$ sample and $\Omega_{2}=13 \pi / 50 \mathrm{rad} / \mathrm{sample}$. With each plant realized in controllable canonical form, we take
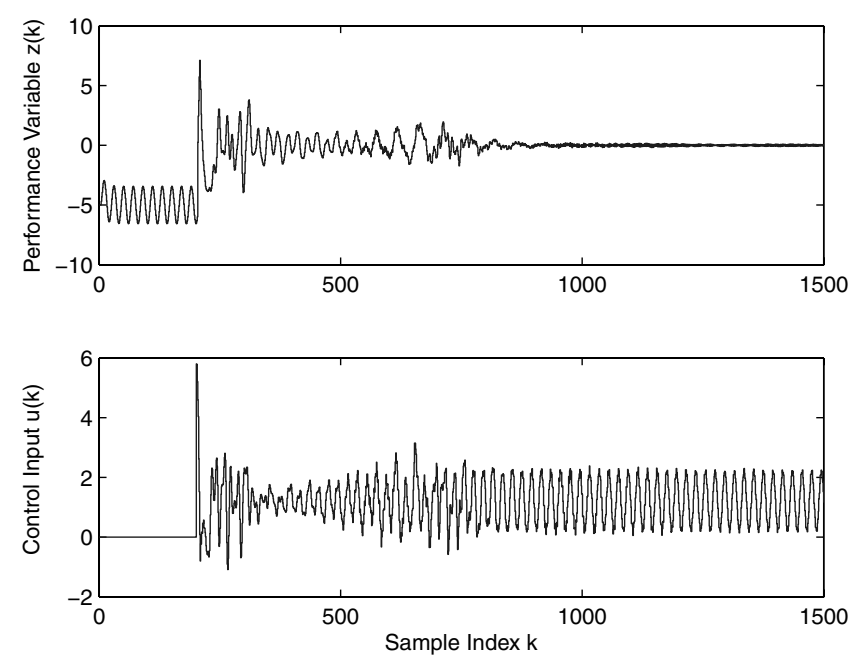

Fig. 7 Closed-loop response of the stable, minimum-phase, SISO plant in Example 6 with a step command and sinusoidal disturbance. The control is turned on at $k=200$. The controller order is $\boldsymbol{n}_{c}=20$ with parameters $p=1, \alpha(k) \equiv 50$, and $r=3$, with $\bar{B}_{z u}$ given by Eq. (하). 

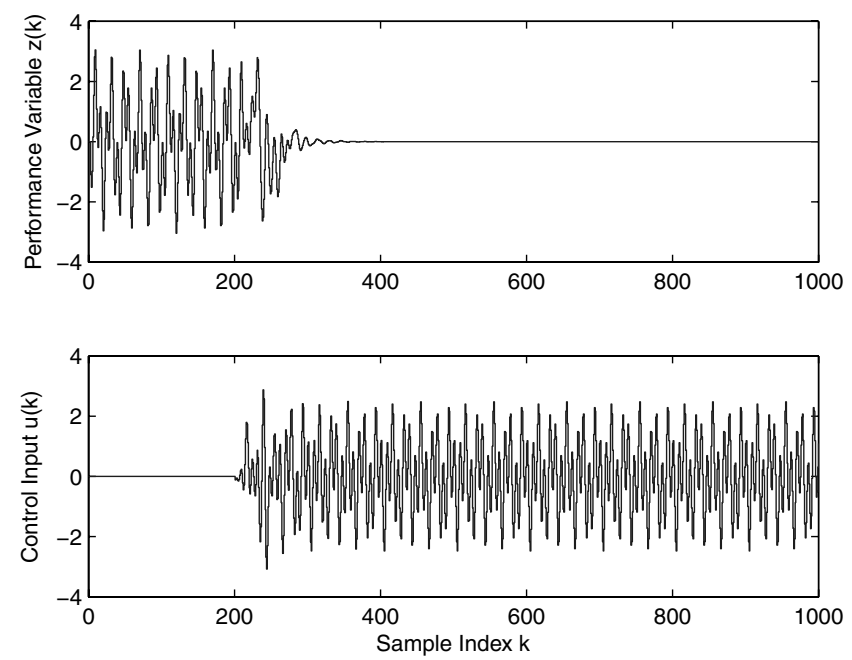

Fig. 8 Closed-loop disturbance-rejection response of the stable, minimum-phase, SISO plant in Example 7. The control is turned on at $k=200$. The controller order is $n_{c}=15$ with parameters $p=1$, $\alpha(k) \equiv 25$, and $r=3$, with $\bar{B}_{z u}$ given by Eq. ( $\left.\underline{56}\right)$.

$$
D_{1}=\left[\begin{array}{c}
I_{2} \\
0
\end{array}\right]
$$

and therefore the disturbance is not matched.

Example 7 (SISO, minimum-phase, stable plant, disturbance rejection). Consider the plant $G_{z u}$ with $d=3$; poles $0.5 \pm 0.5 \mathrm{~J}$, $-0.5 \pm 0.5 \mathrm{~J}, \pm 0.9$, and $\pm 0.7 \mathrm{\jmath}$; and minimum-phase zeros $0.3 \pm 0.7 \jmath,-0.7 \pm 0.3 \jmath$, and 0.5. We take $n_{c}=15, p=1$, $\alpha(k) \equiv 25$, and $r=3$, with $\bar{B}_{z u}$ given by Eq. (56), the closed-loop response is shown in Fig. 8. The control algorithm converges (see Fig. 9) to an internal model controller with high gain at the disturbance frequencies, as shown in Fig. 10.

Example 8 (SISO, non-minimum-phase, stable plant, disturbance rejection). Consider the plant $G_{z u}$ with $d=3$, poles $0.5 \pm 0.5 \mathrm{~J}$, $-0.5 \pm 0.5 \mathrm{~J}, \pm 0.9$, and $\pm 0.7 \mathrm{~J}$; minimum-phase zeros $0.3 \pm 0.7 \mathrm{~J}$ and $-0.7 \pm 0.3 \mathrm{~J}$; and outer non-minimum-phase zero 2 . We take $n_{c}=15, p=1, r=7$, and $\alpha(k) \equiv 25$. The Markov-parameter polynomial used to construct $\bar{B}_{z u}$ as in Eq. (56) is given by

$$
p_{7}(\mathbf{q})=\mathbf{q}^{4}-1.2 \mathbf{q}^{3}-0.96 \mathbf{q}^{2}-0.56 \mathbf{q}-0.75
$$

with roots $0.01 \pm 0.71 \mathrm{~J},-0.77$, and 1.94 . Note that the root 1.94 approximates the zero 2 . The closed-loop response is shown in Fig. 11 .

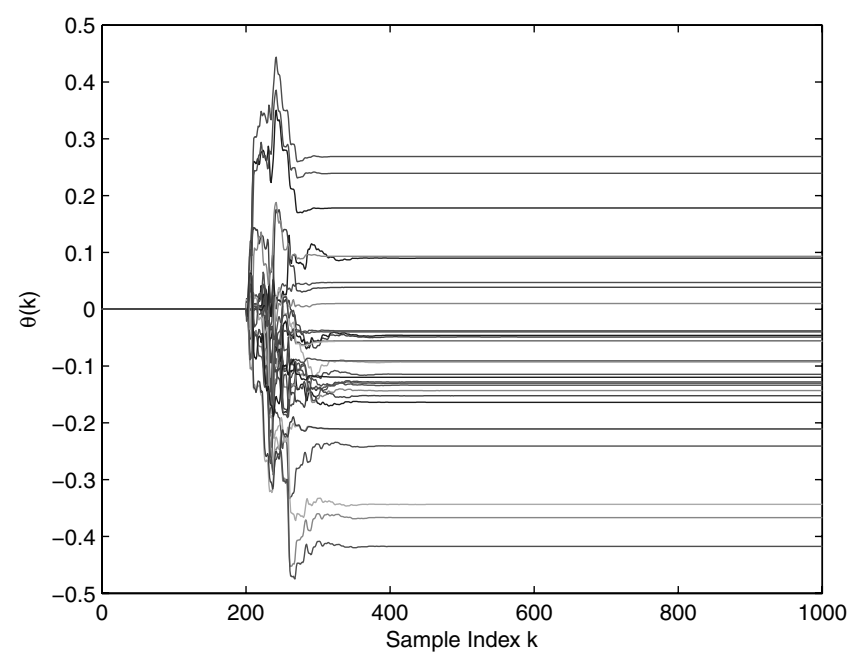

Fig. 9 Time history of the components of $\theta(k)$ for the stable, minimumphase, SISO plant in Example 7. The control is turned on at $k=200$.

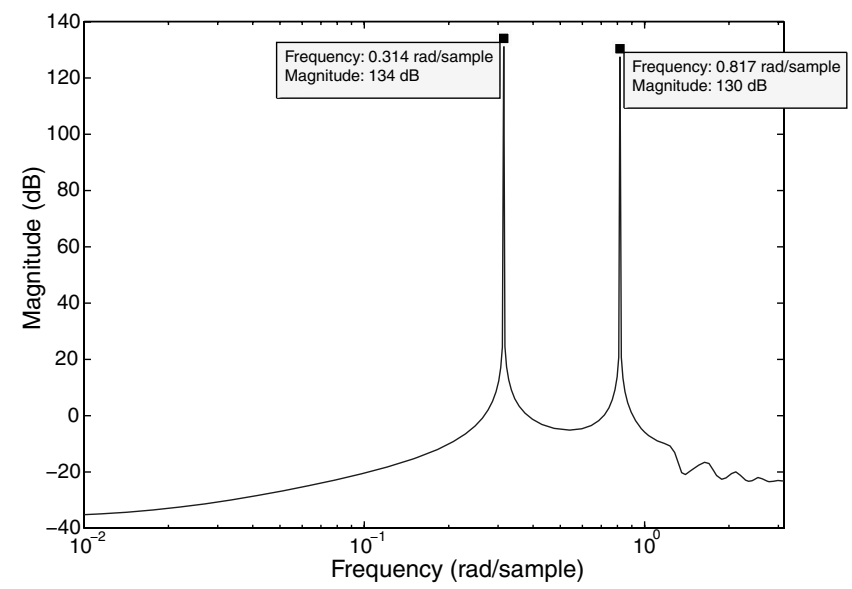

Fig. 10 Bode magnitude plot of the adaptive controller in Example 7 at $k=1000$ samples. The adaptive controller places poles at the disturbance frequencies $\Omega_{1}=\pi / 10 \mathrm{rad} / \mathrm{sample}$ and $\Omega_{2}=13 \pi / 50 \mathrm{rad} / \mathrm{sample}$. The controller magnitude $\left|G_{c}\left(e^{j \Omega}\right)\right|$ is plotted for $\Omega$ up to the Nyquist frequency $\Omega_{\mathrm{Nyq}}=\pi \mathrm{rad} / \mathrm{sample}$.

To illustrate the effect of the learning rate $\alpha(k)$, the closed-loop response is shown in Fig. 12 for $\alpha(k) \equiv 2500$ and all other parameters unchanged. Note that with $\alpha(k) \equiv 2500$, the initial transient is reduced at the expense of convergence speed.

Example 9 (SISO, minimum-phase, unstable plant, disturbance rejection). Consider the plant $G_{z u}$ with $d=3$; poles $0.5 \pm 0.5$ J, $-0.5 \pm 0.5 \mathrm{~J}, \pm 1.04$, and $0.1 \pm 1.025 \mathrm{~J} ;$ and minimum-phase zeros $0.3 \pm 0.7 \mathrm{~J},-0.7 \pm 0.3 \mathrm{~J}$, and 0.5 . We take $n_{c}=15, p=1$, $\alpha(k) \equiv 25$, and $r=10$, with $\bar{B}_{z u}$ given by Eq. (56). The closed-loop response is shown in Fig. 13.

Example 10 (MIMO, minimum-phase, stable plant, disturbance rejection). Consider the two-input, two-output plant

$$
G_{z u}(\mathbf{z})=\left[\begin{array}{cc}
\frac{\mathbf{z}^{2}-0.5 \mathbf{z}}{D_{1}(\mathbf{z})} & \frac{\mathbf{z}^{4}-0.1 \mathbf{z}^{3}-0.22 \mathbf{z}^{2}+0.59 \mathbf{z}-0.29}{D_{1}(\mathbf{z})} \\
\frac{\mathbf{z}-0.5}{D_{1}(\mathbf{z})} & \frac{\mathbf{z}^{3}-1.1 \mathbf{z}^{2}+0.88 \mathbf{z}-0.29}{D_{1}(\mathbf{z})}
\end{array}\right]
$$

where

$$
\begin{gathered}
D_{1}(\mathbf{z}) \triangleq \mathbf{z}^{5}+0.1 \mathbf{z}^{4}+0.09 \mathbf{z}^{3}-0.401 \mathbf{z}^{2}-0.196 \mathbf{z}-0.2205 \\
d=1, \quad H_{1}=\left[\begin{array}{ll}
0 & 1 \\
0 & 0
\end{array}\right]
\end{gathered}
$$

Consequently, $G_{z u}$ has poles $-0.5 \pm 0.5 \jmath, 0.9, \pm 0.7 \jmath,-0.5 \pm 0.5 \mathrm{~J}$, 0.9 , and $\pm 0.7_{J}$ and minimum-phase transmission zeros $0.3 \pm 0.7_{\jmath}$,
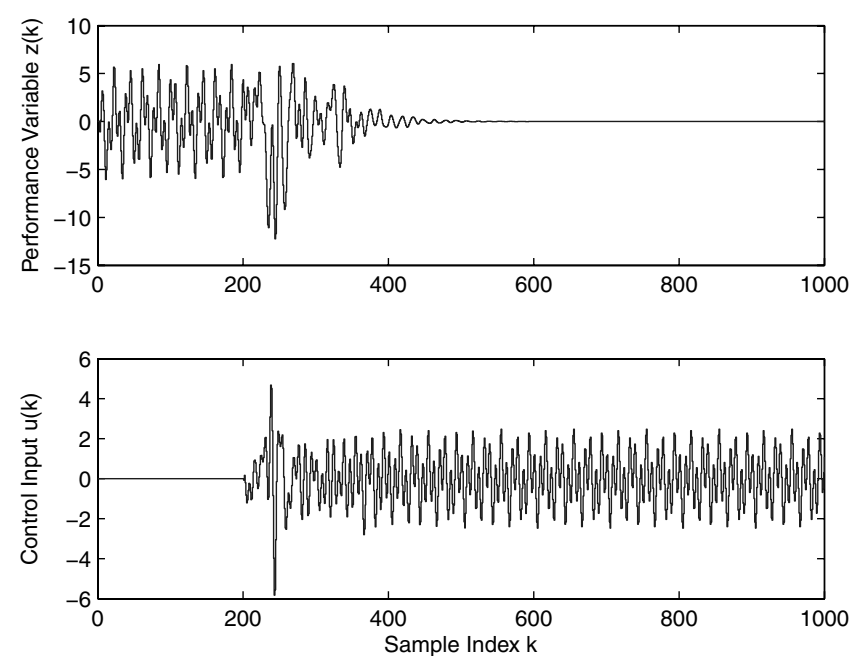

Fig. 11 Closed-loop disturbance-rejection response of the stable, nonminimum-phase, SISO plant in Example 8. The control is turned on at $k=200$. The controller order is $n_{c}=15$ with parameters $p=1$, $\alpha(k) \equiv 25$, and $r=7$, with $\bar{B}_{z u}$ given by Eq. ( $\underline{56}$. 

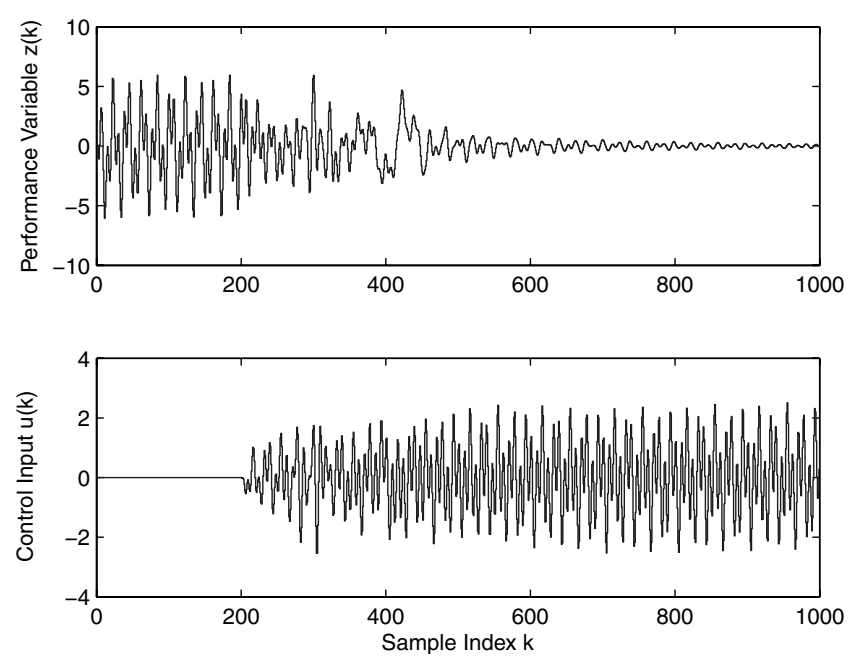

Fig. 12 Closed-loop disturbance-rejection response of the stable, nonminimum-phase, SISO plant in Example 8. The control is turned on at $k=200$. The controller order is $n_{c}=15$ with parameters $p=1$, $\alpha(k) \equiv 2500$, and $r=7$ with $\bar{B}_{z u}$ given by Eq. (56). Compared with Fig. 11, the initial transient is reduced at the expense of convergence speed.

0.5 , and 0.5 . We take $n_{c}=15, p=1, \alpha(k) \equiv 1$, and $r=10$, with $\bar{B}_{z u}$ given by Eq. (56). The closed-loop response is shown in Fig. 14.

Example 11 MIMO, non-minimum-phase, stable plant, disturbance rejection). Consider the two-input, two-output plant

$$
G_{z u}(\mathbf{z})=\left[\begin{array}{cc}
\frac{\mathbf{z}^{2}-0.5 \mathbf{z}}{D_{1}(\mathbf{z})} & \frac{\mathbf{z}^{2}-\mathbf{z}-2}{D_{2}(\mathbf{z})} \\
\frac{\mathbf{z}-0.5}{D_{1}(\mathbf{z})} & \frac{\mathbf{z}-2}{D_{2}(\mathbf{z})}
\end{array}\right]
$$

where $D_{1}(\mathbf{z})$ is given in Example 10,

$$
\begin{gathered}
D_{2}(\mathbf{z}) \triangleq \mathbf{z}^{3}-0.2 \mathbf{z}^{2}+0.34 \mathbf{z}+0.232, \quad d=1 \\
H_{1}=\left[\begin{array}{ll}
0 & 1 \\
0 & 0
\end{array}\right]
\end{gathered}
$$

Consequently, $G_{z u}$ has poles $-0.5 \pm 0.5 \jmath, 0.3 \pm 0.7 \jmath, \pm 0.7 \jmath,-0.4$, and 0.9 ; minimum-phase transmission zero 0.5 ; and outer nonminimum-phase transmission zero 2. We take $n_{c}=15, p=2$, $\alpha(k) \equiv 1$, and $r=8$, with $\bar{B}_{z u}$ given by Eq. ( $\left.\underline{56}\right)$. The closed-loop response is shown in Fig. 15.
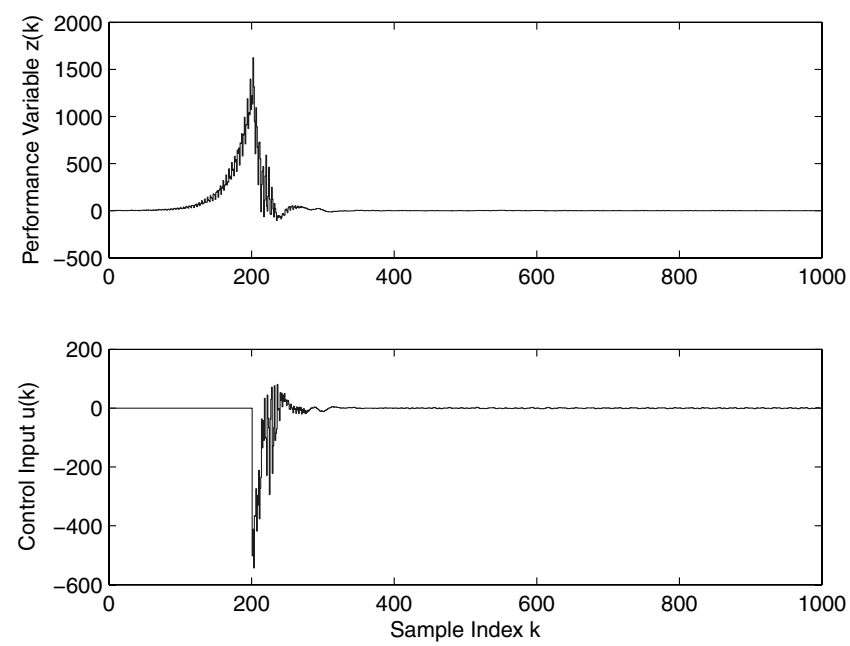

Fig. 13 Closed-loop disturbance-rejection response of the unstable, minimum-phase, SISO plant in Example 9. The control is turned on at $k=200$. The controller order is $n_{c}=15$ with parameters $p=1$, $\alpha(k) \equiv 25$, and $r=10$, with $\bar{B}_{z u}$ given by Eq. (56).
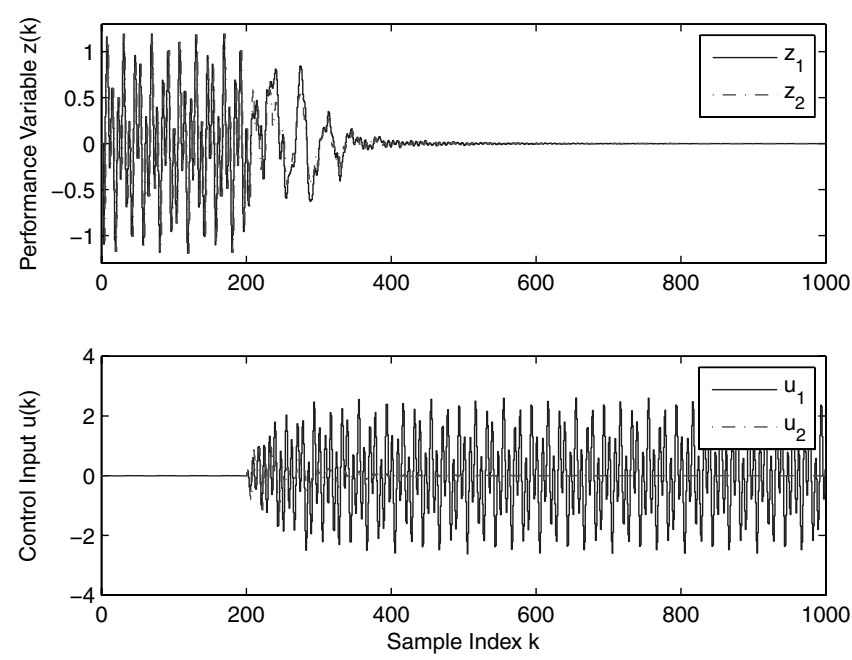

Fig. 14 Closed-loop disturbance-rejection response of the stable, minimum-phase, two-input, two-output plant in Example 10. The control is turned on at $k=200$. The controller order is $n_{c}=15$ with parameters $p=1, \alpha(k) \equiv 1$, and $r=10$, with $\bar{B}_{z u}$ given by Eq. ( $\left.\underline{56}\right)$.

Example 12 (MIMO, non-minimum-phase, unstable plant, disturbance rejection). Consider the two-input, two-output plant

$$
G_{z u}(\mathbf{z})=\left[\begin{array}{cc}
\frac{\mathbf{z}^{2}-0.5 \mathbf{z}}{D_{3}(\mathbf{z})} & \frac{\mathbf{z}^{2}-\mathbf{z}-2}{D_{4}(\mathbf{z})} \\
\frac{\mathbf{z}-0.5}{D_{3}(\mathbf{z})} & \frac{\mathbf{z}-2}{D_{4}(\mathbf{z})}
\end{array}\right]
$$

where

$$
\begin{gathered}
D_{3}(\mathbf{z}) \triangleq \mathbf{z}^{5}-1.1 \mathbf{z}^{4}+1.731 \mathbf{z}^{3}-1.494 \mathbf{z}^{2}+0.608 \mathbf{z}-0.4679 \\
D_{4}(\mathbf{z}) \triangleq \mathbf{z}^{3}+1.4 \mathbf{z}^{2}+0.9 \mathbf{z}+0.2, \quad d=1, \quad H_{1}=\left[\begin{array}{ll}
0 & 1 \\
0 & 0
\end{array}\right]
\end{gathered}
$$

Consequently, $G_{z u}$ has poles $-0.5 \pm 0.5 \jmath, \pm 0.7 \jmath, 0.1 \pm 1.025 \mathrm{~J}$, -0.4 , and 0.9 ; minimum-phase transmission zero 0.5 ; and outer non-minimum-phase transmission zero 2 . We take $n_{c}=10, p=1$, $\alpha(k) \equiv 1$, and $r=10$, with $\bar{B}_{z u}$ given by Eq. ( $\left.\underline{56}\right)$. The closed-loop response is shown in Fig. 16.

\section{Numerical Examples: Offnominal Cases}

We now revisit the numerical examples of Sec. VII to illustrate the response of the RCF adaptive control algorithm under conditions of
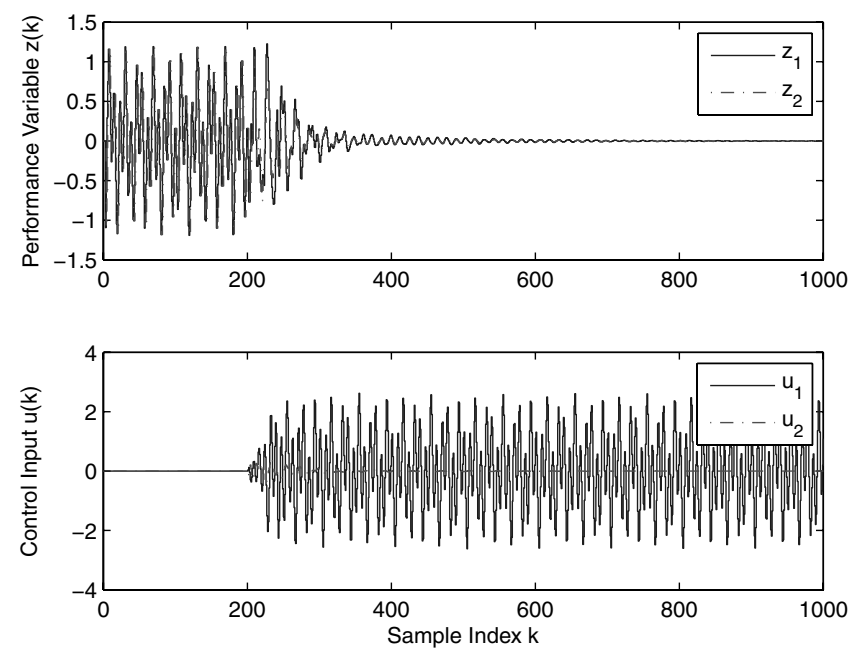

Fig. 15 Closed-loop disturbance-rejection response of the stable, nonminimum-phase, two-input, two-output plant in Example 11. The control is turned on at $k=200$. The controller order is $n_{c}=15$ with parameters $p=2, \alpha(k) \equiv 1$, and $r=8$, with $\bar{B}_{z u}$ given by Eq. ( $\left.\underline{56}\right)$. 

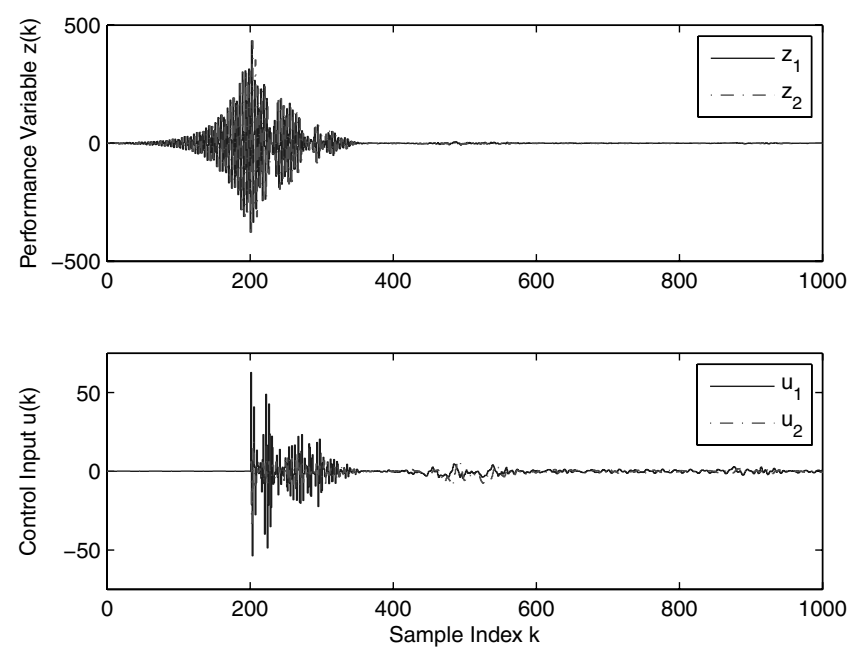

Fig. 16 Closed-loop disturbance-rejection response of the unstable, non-minimum-phase, two-input, two-output plant in Example 12. The control is turned on at $k=200$. The controller order is $n_{c}=10$ with parameters $p=1, \alpha(k) \equiv 1$, and $r=10$, with $\bar{B}_{z u}$ given by Eq. (하).

uncertainty in the relative degree and Markov parameters as well as measurement noise and actuator and sensor saturation. In each example, the adaptive controller gain matrix $\theta(k)$ is initialized to zero. Unless otherwise noted, all examples assume $x(0)=0$.

Example 13 (Example 8 with Markov-parameter multiplicative error). Reconsider Example 8 with Markov-parameter multiplicative error. For controller implementation, we use the estimate $\hat{B} \triangleq \eta B$, where $\eta \in \mathbb{R}$ is varied between 0.3 and 5 . For $i=1, \ldots, r$, the estimated Markov parameters $\hat{H}_{i}=C A^{i-1} \hat{B}$ are used to construct $\bar{B}_{z u}$ given by Eq. (56). Taking $n_{c}=15, p=1, r=10$, and $\alpha(k) \equiv 1000$, the closed-loop performance is compared in Fig. 17. In each case, the control is turned on at $k=0$, and the performance metric is given by

$$
k_{0} \triangleq \min \left\{k \geq 9: \frac{1}{10} \sum_{i=0}^{9}|z(k-i)|<0.01\right\}
$$

that is, $k_{0}$ is the minimum time step $k$ such that the average of $\{|z(k-i)|\}_{i=0}^{9}$ is less than 0.01 . Figure 17 shows that the best performance is obtained for $\eta=1$, which corresponds to the true value of $B$. As $\eta$ is decreased, convergence slows significantly. In the

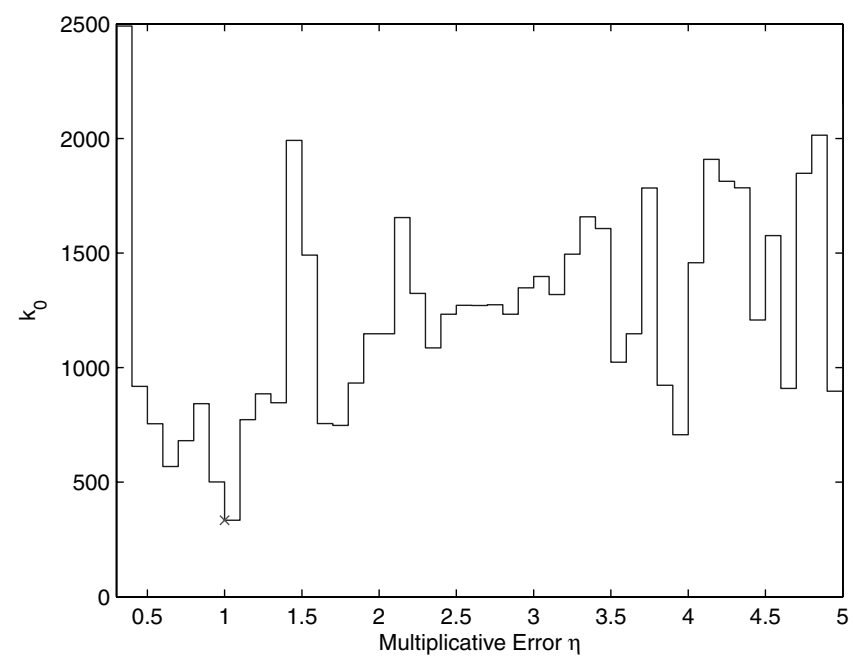

Fig. 17 Closed-loop performance comparison of the stable, nonminimum-phase, SISO plant in Example 8 with multiplicative error in $B$. We take $n_{c}=10, p=1$, and $\alpha(k) \equiv 1000$. The multiplicative error $\eta$, which is used to obtain the Markov parameters for $\bar{B}_{z u}$ given by Eq. (56) with $r=10$, is varied between 0.3 and 5 . The best performance is obtained for $\eta=1$, which corresponds to the true value of $B$.
Table 2 Closed-loop performance comparison of the stable, non-minimum-phase, SISO plant in Example 8 with unknown latency

\begin{tabular}{lcc}
\hline \hline$\hat{d^{\mathrm{a}}}$ & $k_{0}$ & $\max |z(k)|$ \\
\hline 2 & 1870 & 12.3 \\
3 & 531 & 9.4 \\
4 & 847 & 8.5 \\
5 & 4633 & 10.9 \\
6 & 11,660 & $3.2 \times 10^{9}$ \\
\hline \hline
\end{tabular}

${ }^{\text {aFor controller implementation, we use the erroneous estimate } \hat{d}}$ of $d$ and take $n_{c}=15, p=1, \alpha(k) \equiv 1000$, and $r=10$, with $\bar{B}_{z u}$ given by Eq. (56). The best performance is obtained for $d=d=3$.

case in which the sign of the first nonzero Markov parameter (the sign of the high-frequency gain) is wrong, that is, $\hat{H}_{3}=-H_{3}$, the simulation fails. These simulations suggest that performance degradation due to an unknown scaling of the Markov parameters provides a useful measure of adaptive gain margin. These findings are consistent with the adaptive gain-margin results presented in [3].

Example 14 (Example 8 with unknown latency). A known latency of $l$ steps can be accounted for by replacing $d$ by $d+l$ in the construction of $\bar{B}_{z u}$. However, we now assess the effect of unknown latency in Example 8, which is equivalent to uncertainty in the relative degree $d$. The system has relative degree $d=3$. For controller implementation, we use the erroneous estimate $\hat{d}$ of $d$ and take $n_{c}=15, p=1, \alpha(k) \equiv 1000$, and $r=10$, with $\bar{B}_{z u}$ given by Eq. (56). Letting $\hat{d}$ be either $2,3,4,5$, or 6 , Table 2 compares both the performance metric (59) and the maximum value of $|z(k)|$ for each estimate $\hat{d}$ of $d$. In each case, the control is turned on at $k=0$. The best performance is obtained for $\hat{d}=d=3$. These simulations show the sensitivity of the adaptive controller to unknown errors in the relative degree $d$, which provides a useful measure of adaptive phase margin.

Example 15 (sensitivity to non-minimum-phase-zero uncertainty). Consider the plant $G_{z u}$ with $d=1 ; H_{1}=1$; poles 0 and 0.5 ; and outer non-minimum-phase zero 2 . The plant is subject to disturbance $w(k)$ given by Eq. (58); thus, with the plant realized in controllable canonical form, we take $D_{1}=I_{2}$ and $E_{0}=0$. Furthermore, we assume $y=z$ and let $\phi(k)$ be given by Eq. (37). To illustrate the sensitivity of the adaptive control algorithm to knowledge of the nonminimum-phase zero, we let $\bar{B}_{z u}$ be given by Eq. ( $\underline{51}$ ), which is constructed using the first nonzero Markov parameter $H_{1}=1$, the

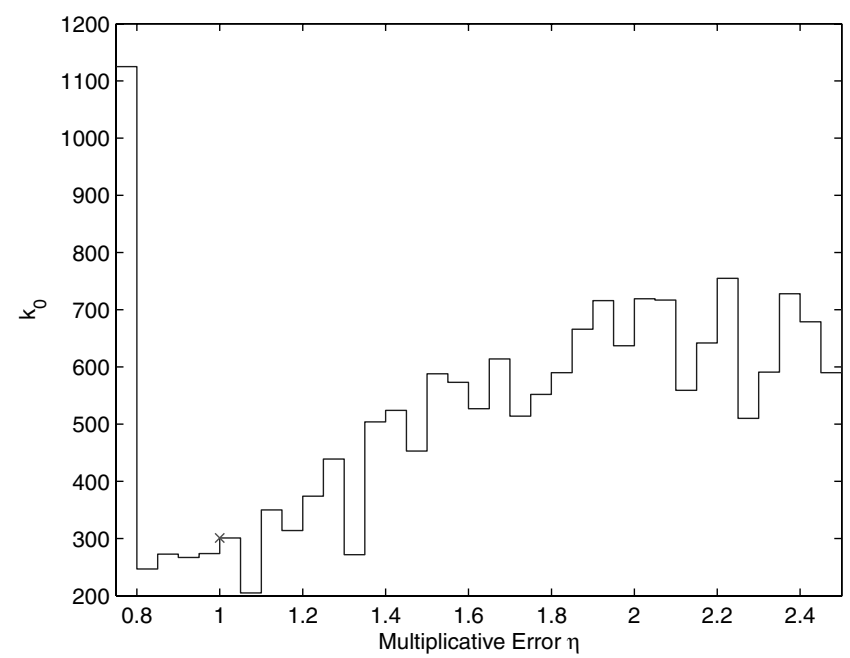

Fig. 18 Closed-loop performance comparison of the stable, nonminimum-phase, SISO plant in Example 15 with a multiplicative error in the non-minimum-phase zero 2 . We take $n_{c}=10, p=1$, and $\alpha(k) \equiv 25$. The non-minimum-phase-zero multiplicative error $\eta$, which is used to construct $\bar{B}_{z u}$ given by Eq. (51), is varied between 0.75 and 2.5 . The best performance is obtained for $\eta=1.05$, which is close to the true value of the non-minimum-phase zero. 

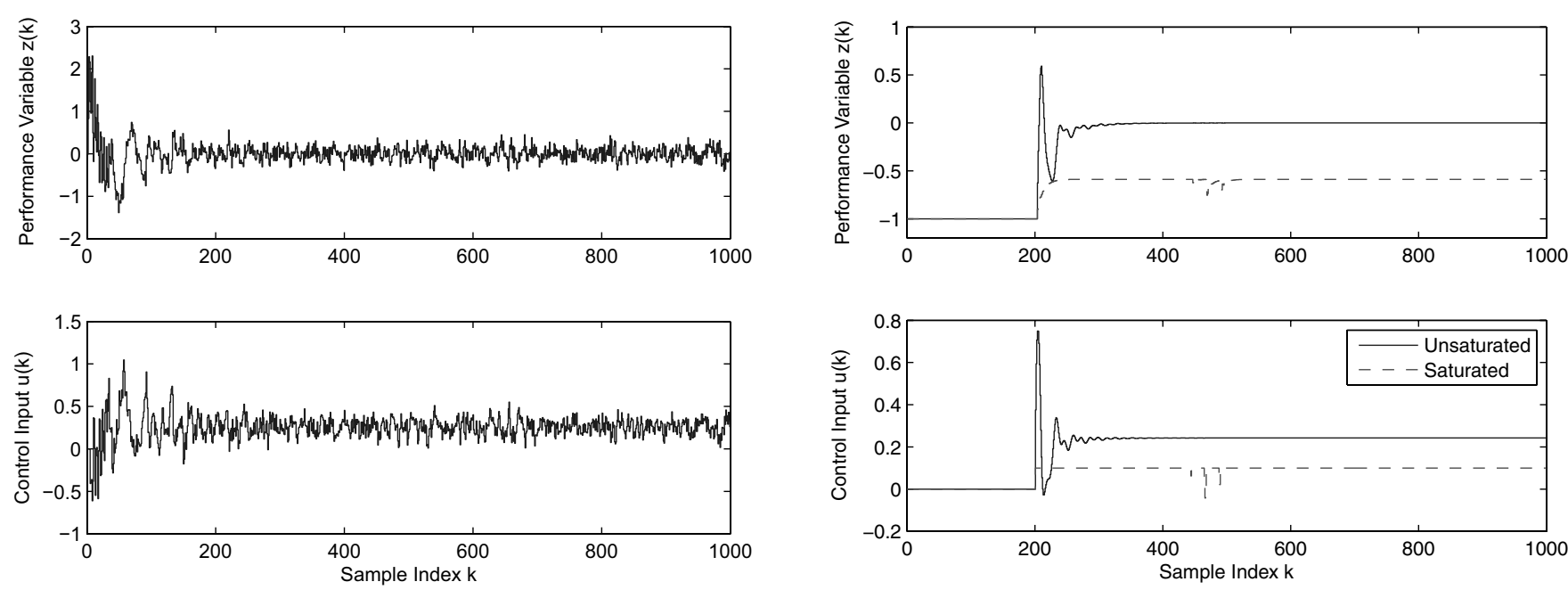

Fig. 19 Closed-loop response of the unstable, minimum-phase, SISO plant in Example 9 with random white noise added to the measurement. The control is turned on at $k=0$. The controller order is $n_{c}=15$ with parameters $p=1, \alpha(k) \equiv 25$, and $r=3$, with $\bar{B}_{z u}$ given by Eq. (56). The performance variable is degraded to the level of the additive sensor noise $v(k)$.

non-minimum-phase zero 2 , and a multiplicative error $\eta \in \mathbb{R}$ : that is, $N(\mathbf{q})=\mathbf{q}-2 \eta$. We vary $\eta$ between 0.75 and 2.5 with $n_{c}=10$, $p=1$, and $\alpha(k) \equiv 25$. A closed-loop performance comparison is shown in Fig. 18. In each case, the control is turned on at $k=0$, and the performance metric is given by Eq. (59). The best performance is obtained for $\eta=1.05$, which is close to the true value of the nonminimum-phase zero. Note that the adaptive control algorithm is more robust to larger values of $\eta$ than smaller values.

Example 16 (Example 9 with stabilization and noisy measurements). Reconsider Example 9 with no commands or disturbances. For stabilization, we take $D_{1}$ and $E_{0}$ to be zero matrices. To assess the performance of the adaptive algorithm with added sensor noise, we modify Eqs. (2) and (3) by

$$
y(k)=z(k)=E_{1} x(k)+E_{0} w(k)+v(k)
$$

where $v(k) \in \mathbb{R}^{l_{z}}$ is Gaussian white noise with mean $\bar{v}=2$ and standard deviation $\sigma=0.1$. We take $n_{c}=15, p=1, \alpha(k) \equiv 25$, and $r=3$, with $\bar{B}_{z u}$ given by Eq. ( $\underline{56}$ ). For the initial condition
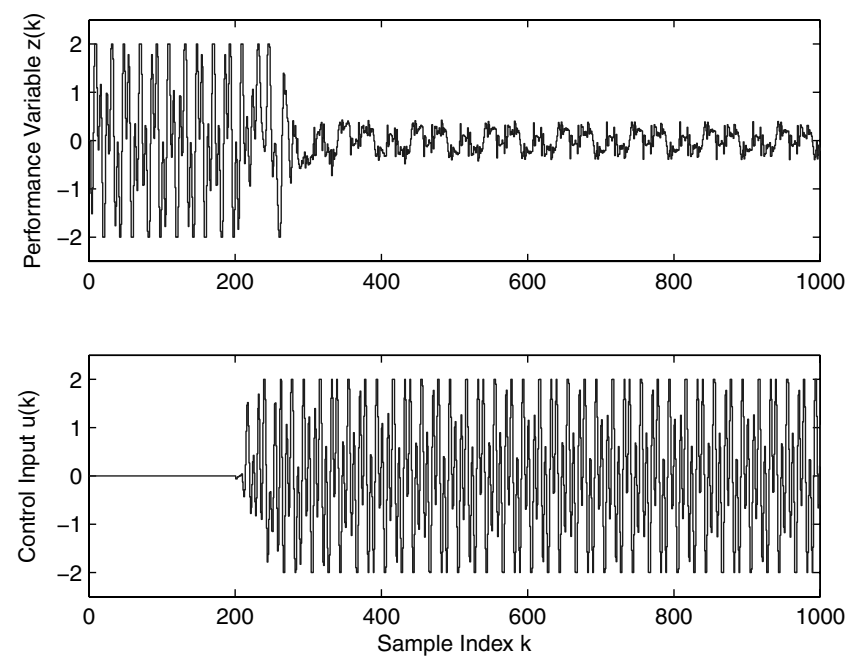

Fig. 20 Closed-loop disturbance-rejection response of the stable, minimum-phase, SISO plant in Example 7, where both the actuator and sensor are saturated at \pm 2 . The control is turned on at $k=200$. The controller order is $n_{c}=15$ with parameters $p=1, \alpha(k) \equiv 25$, and $r=3$, with $\bar{B}_{z u}$ given by Eq. ( $\underline{56}$ ). The saturations degrade steady-state performance.

Fig. 21 Closed-loop step-command-following responses of the stable, minimum-phase, SISO plant in Example 7 with and without actuator saturation at \pm 0.1 . The control is turned on at $k=200$. The controller order is $n_{c}=15$ with parameters $p=1, \alpha(k) \equiv 25$, and $r=3$, with $\bar{B}_{z u}$ given by Eq. (56).

$$
x(0)=\left[\begin{array}{llllllll}
-0.43 & -1.67 & 0.13 & 0.29 & -1.15 & 1.19 & 1.19 & -0.04
\end{array}\right]^{T}
$$

the closed-loop response is shown in Fig. 19.

Example 17 (Example 7 with actuator and sensor saturation). Reconsider Example 7 with the additional assumption that both the control input and sensor measurement are subject to saturation at \pm 2 . We take $n_{c}=15, p=1, \alpha(k) \equiv 25$, and $r=3$, with $\bar{B}_{z u}$ given by Eq. (하). The closed-loop response shown in Fig. 20 indicates that the saturations degrade steady-state performance.

Example 18 (Example 7 with command-following and actuator saturation). Reconsider Example 7 with step command given by $w(k)=1$. With the plant realized in controllable canonical form, we take $D_{1}=0$ and $E_{0}=-1$. Taking $n_{c}=15, p=1, \alpha(k) \equiv 25$, and $r=3$, with $\bar{B}_{z u}$ given by Eq. (56), the closed-loop responses are shown in Fig. 21 with and without actuator saturation at \pm 0.1 . With actuator saturation, the performance variable reflects the capability of the saturated control.

\section{Model Reference Adaptive Control}

Model reference adaptive control (MRAC), as illustrated in Fig. 22, is a special case of Eqs. (1-3), where $z \triangleq y_{1}-y_{m}$ is the difference between the measured output $y_{1}$ of the plant $G$ and the output $y_{m}$ of a reference model $G_{m}$. For MRAC, the exogenous command $w$ is assumed to be available to the controller as an additional measurement variable $y_{2}$. Unlike standard MRAC methods [1,7,16,31-33], retrospective cost adaptive control does not depend on knowledge of the reference model $G_{m}$.

We now present numerical examples to illustrate the response of the RCF adaptive control algorithm for model reference adaptive

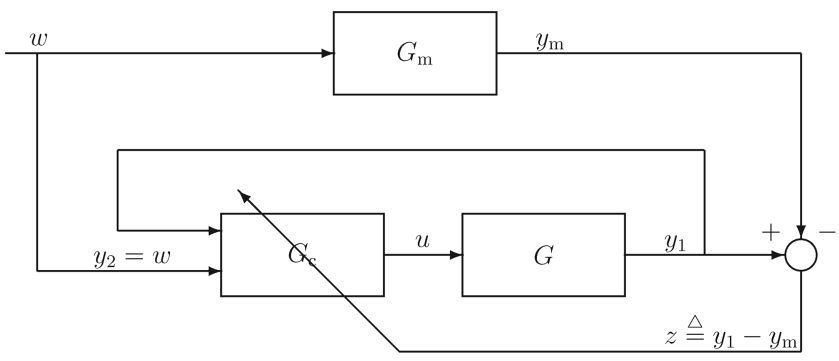

Fig. 22 Model reference adaptive control problem with performance variable $z$. 

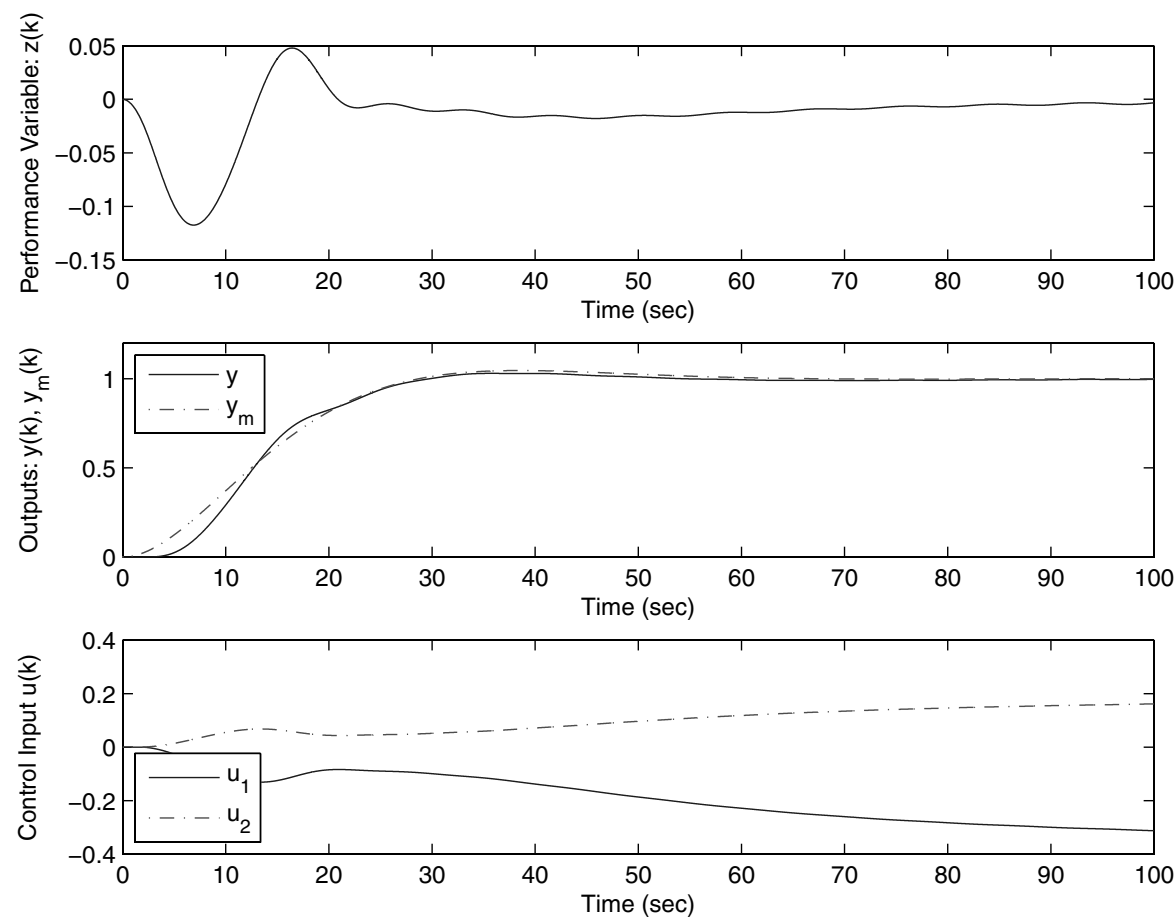

Fig. 23 Closed-loop model reference adaptive control of Boeing 747 longitudinal dynamics. The controller order is $n_{c}=10$ with parameters $p=1$, $\alpha(k) \equiv 40$, and $r=10$, with $\bar{B}_{z u}$ given by Eq. (56). The controller is turned on at $t=0 \mathrm{~s}$, and the performance variable converges within about $20 \mathrm{~s}$.

control (see Fig. 22). Unless otherwise noted, the adaptive controller gain matrix $\theta(k)$ is initialized to zero.

\section{A. Boeing $\mathbf{7 4 7}$ Longitudinal Dynamics}

Consider the longitudinal dynamics of a Boeing 747 aircraft, linearized about steady flight at $40,000 \mathrm{ft}$ and $774 \mathrm{ft} / \mathrm{s}$. The inputs to the dynamical system are taken to be elevator deflection and thrust, and the output is the pitch angle. The continuous-time equations of motion are thus given by

$$
\begin{aligned}
& {\left[\begin{array}{c}
\dot{u} \\
\dot{w} \\
\dot{q} \\
\dot{\theta}
\end{array}\right]=\left[\begin{array}{cccc}
-0.003 & 0.039 & 0 & -0.322 \\
-0.065 & -0.319 & 7.74 & 0 \\
0.020 & -0.101 & -0.429 & 0 \\
0 & 0 & 1 & 0
\end{array}\right]\left[\begin{array}{l}
u \\
w \\
q \\
\theta
\end{array}\right]} \\
& +\left[\begin{array}{cc}
0.010 & 1 \\
-0.180 & -0.040 \\
-1.160 & 0.598 \\
0 & 0
\end{array}\right]\left[\begin{array}{l}
\delta_{e} \\
\delta_{T}
\end{array}\right]
\end{aligned}
$$
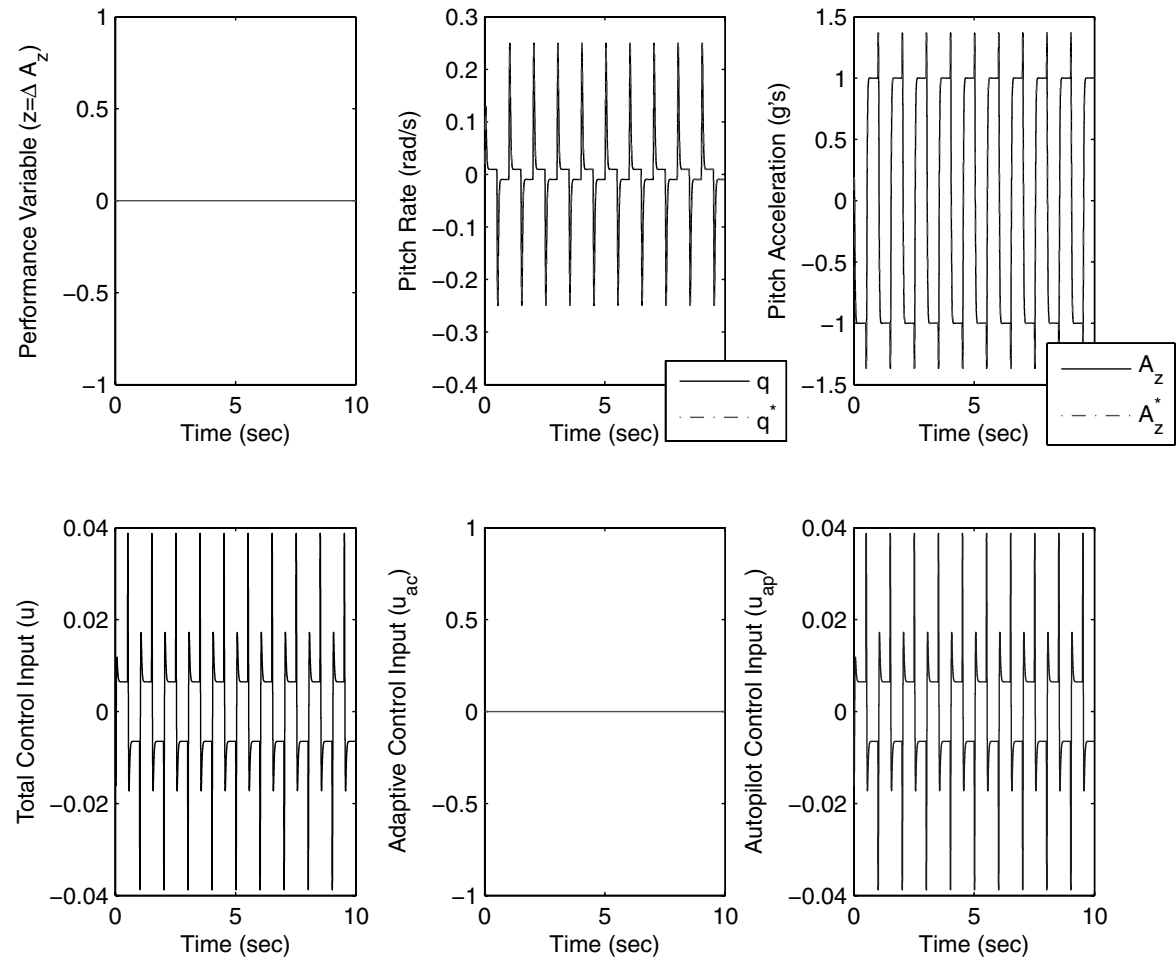

Fig. 24 Closed-loop model reference adaptive control of missile longitudinal dynamics. The control effectiveness $\lambda=1$, and thus the plant and reference model are identical. Therefore, the adaptive control input $u_{\mathrm{ac}}=\mathbf{0}$. 

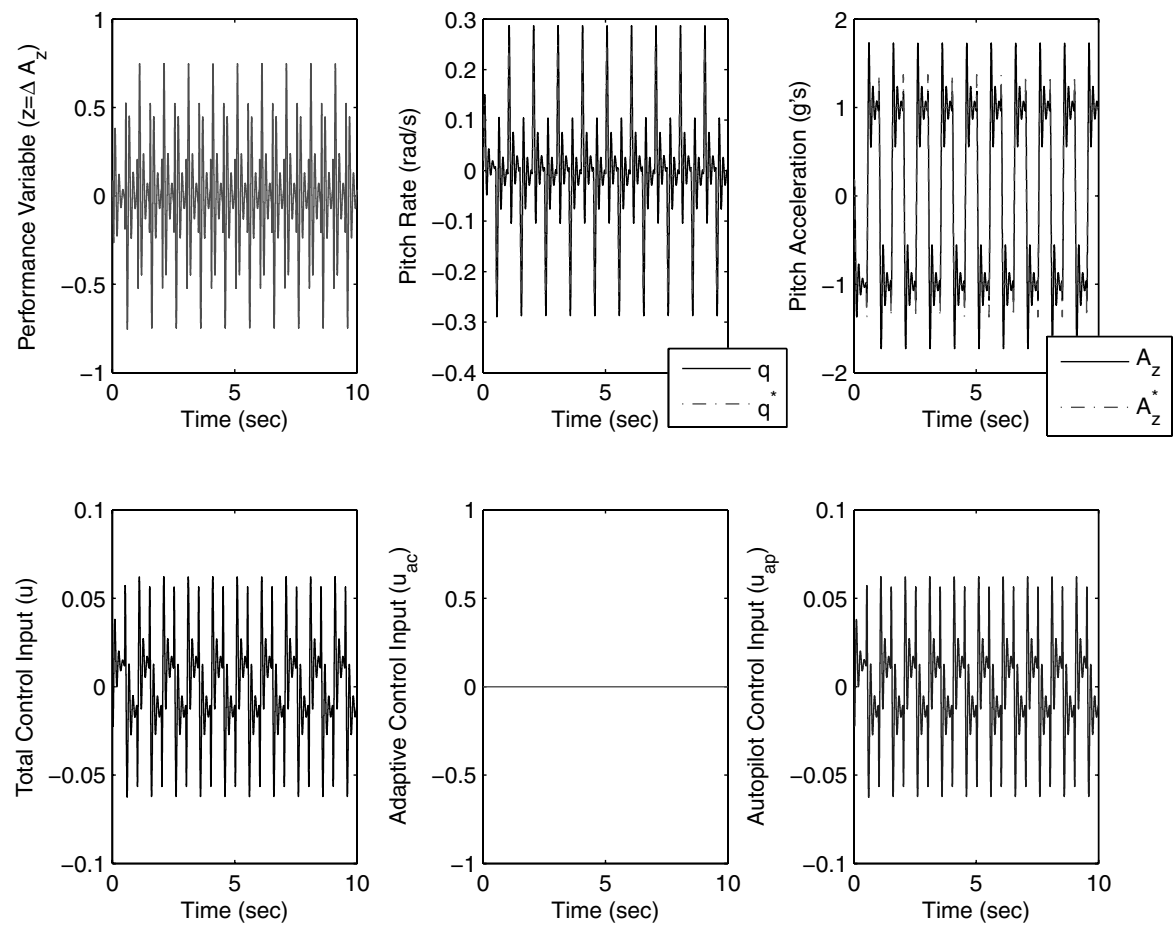

Fig. 25 Missile longitudinal dynamics with control effectiveness $\lambda=0.50$ and adaptive controller turned off: that is, autopilot-only control.

$$
\begin{gathered}
y=\left[\begin{array}{l}
y_{1} \\
y_{2}
\end{array}\right]=\left[\begin{array}{llll}
0 & 0 & 0 & 1 \\
0 & 0 & 0 & 0
\end{array}\right]\left[\begin{array}{l}
u \\
w \\
q \\
\theta
\end{array}\right]+\left[\begin{array}{l}
0 \\
1
\end{array}\right] w \\
z=y_{1}-y_{m}
\end{gathered}
$$

where $w$ is the exogenous command and $y_{m}$ is the output of the reference model:

$$
G_{m}(s)=\frac{Y_{m}(s)}{W(s)}=\frac{0.0131}{s^{2}+0.16 s+0.0131}
$$

We discretize Eqs. (61-64) using a zero-order hold and sampling time $T_{s}=0.01 \mathrm{~s}$. The reference command is taken to be a $1 \mathrm{deg}$ step command in pitch angle. The controller order is $n_{c}=10$ with parameters $p=1, \alpha(k) \equiv 40$, and $r=10$, with $\bar{B}_{z u}$ given by Eq. (56). The closed-loop response is shown in Fig. 23 for zero initial conditions.

\section{B. Missile Longitudinal Dynamics}

We now present numerical examples for MRAC of missile longitudinal dynamics under offnominal or damage situations. The missile longitudinal plant [34] is derived from the short-period approximation of the longitudinal equations of motion, given by
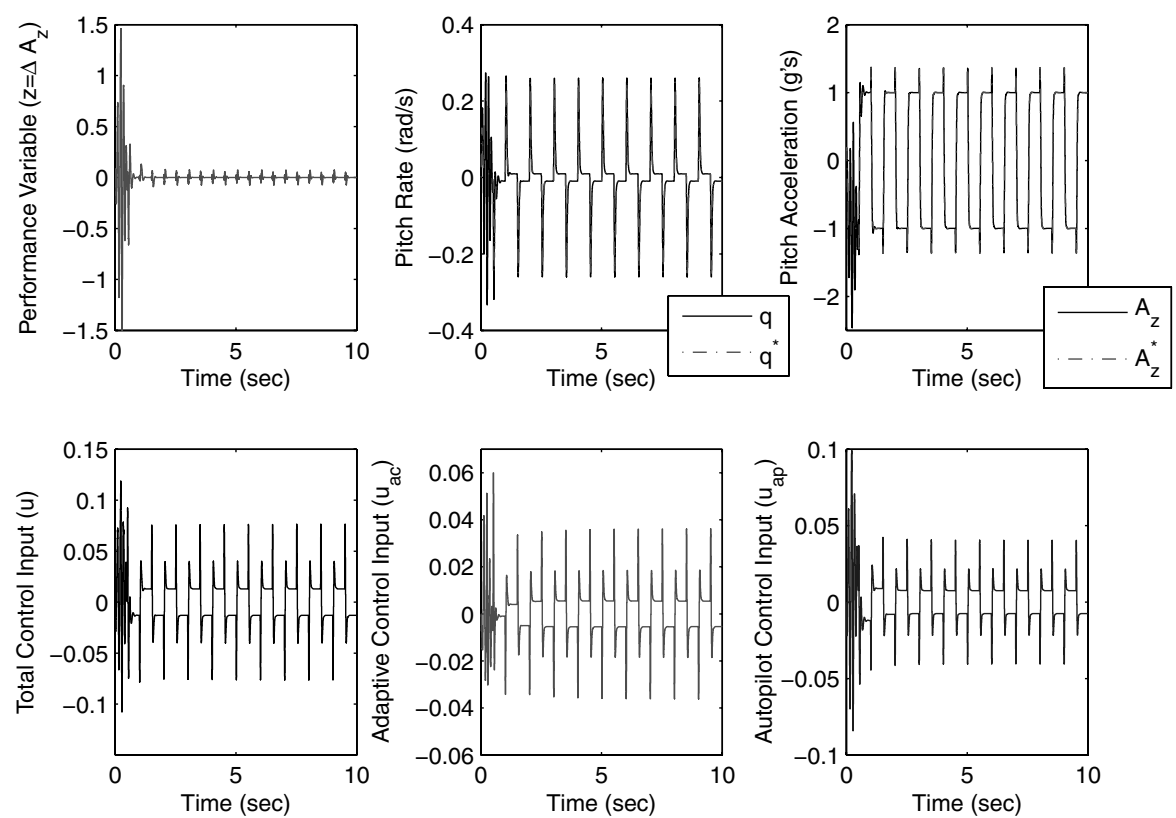

Fig. 26 Closed-loop model reference adaptive control of missile longitudinal dynamics with control effectiveness $\lambda=0.50$. The augmented controllers provide better performance than the autopilot-only simulation. 

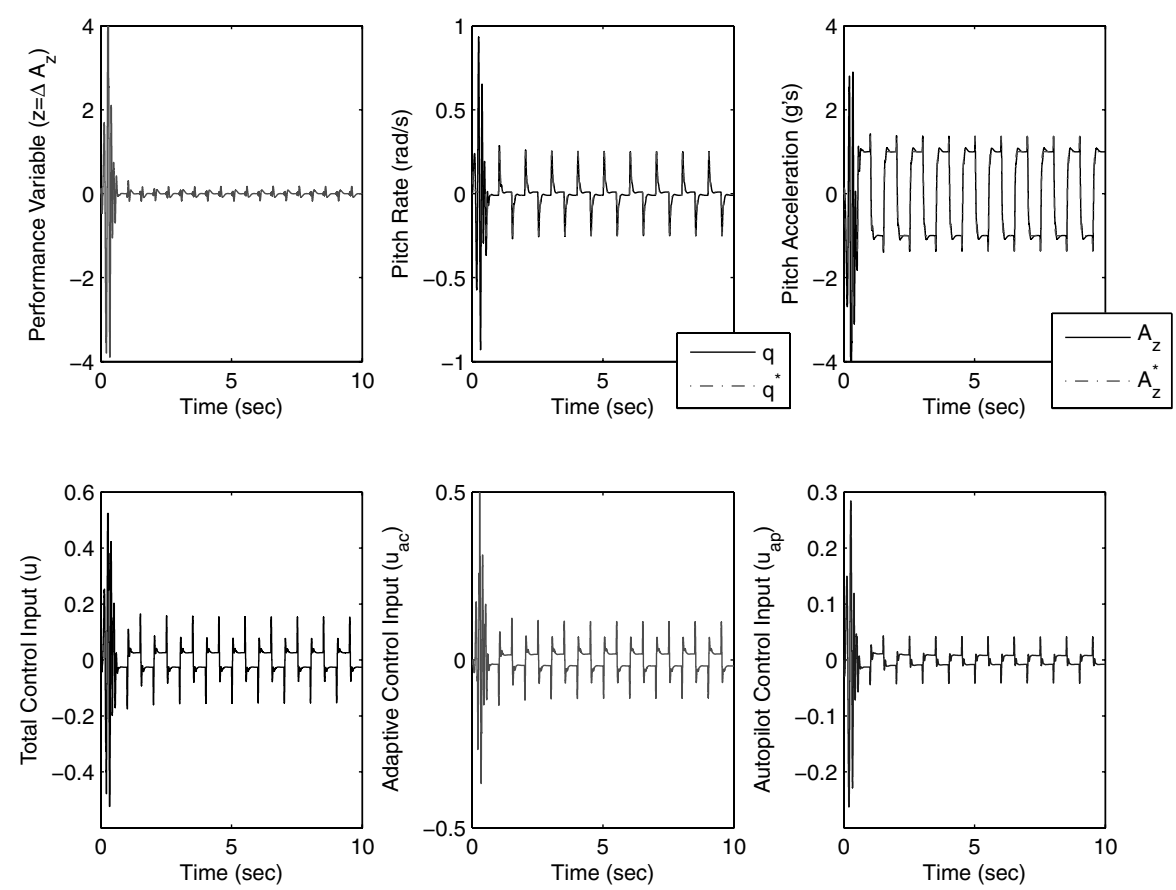

Fig. 27 Closed-loop model reference adaptive control of missile longitudinal dynamics with control effectiveness $\lambda=0.25$. After a transient, the augmented controllers stabilize the system, whereas the autopilot-only simulation fails. Note that the system is stabilized despite the total control input $u$ reaching the actuator saturation level of $\pm 30 \mathrm{deg}$.

$$
\begin{aligned}
& \dot{x}=\left[\begin{array}{cc}
-1.064 & 1 \\
290.26 & 0
\end{array}\right] x+\lambda\left[\begin{array}{c}
-0.25 \\
-331.4
\end{array}\right] u \\
& y=\left[\begin{array}{cc}
-123.34 & 0 \\
0 & 1
\end{array}\right] x+\lambda\left[\begin{array}{c}
-13.51 \\
0
\end{array}\right] u
\end{aligned}
$$

where
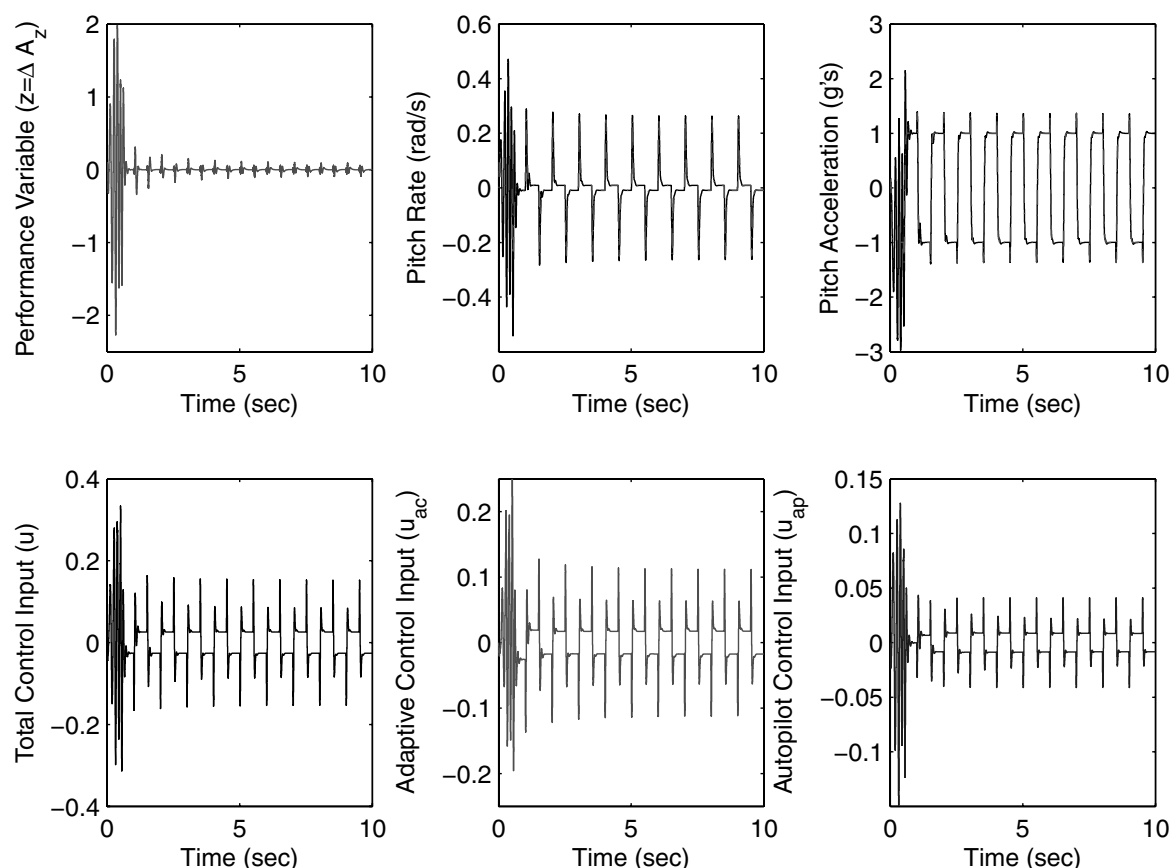

Fig. 28 Closed-loop model reference adaptive control of missile longitudinal dynamics with control effectiveness $\lambda=0.25$. The adaptive controller is initialized with the converged gains from the $50 \%$ control effectiveness case. The initial transient is reduced as compared with initializing the control gains to zero. In this case, the actuator saturation level is never reached. 
of the basic missile longitudinal plant with $\lambda=1$ and the classical three-loop autopilot. An actuator amplitude saturation of $\pm 30 \mathrm{deg}= \pm 0.524 \mathrm{rad}$ is included in the model, but no actuator or sensor dynamics are included.

The goal is to have the missile follow a pitch acceleration command $w$ consisting of a $1 \mathrm{~g}$ amplitude, $1 \mathrm{~Hz}$ square wave. The performance variable $z$ is the difference between the measured pitch acceleration $A_{z}$ and the reference model pitch acceleration $A_{z}^{*}$ : that is, $z \triangleq A_{z}-A_{z}^{*}$. The closed-loop response is shown in Fig. 24 for $\lambda=1$. Since the plant and reference model are identical in the nominal case, the adaptive control input $u_{\mathrm{ac}}=0$.

All of the following examples use zero initial conditions and the same adaptive controller parameters. The adaptive controller is implemented at a sampling rate of $300 \mathrm{~Hz}$. We take $n_{c}=3, p=1$, and $r=20$, with $\bar{B}_{z u}$ given by Eq. (56). A time-varying learning rate $\alpha(k)=75 k+1$ is used such that, initially, controller adaptation is fast and as performance improves, the adaptation slows. The learning rate is identical for each simulation. System identification using the observer/Kalman filter identification algorithm [30] is used to obtain the 20 Markov parameters required for controller implementation. The offline identification procedure is performed with a nominal simulation $(\lambda=1)$ by injecting band-limited white noise at the adaptive controller input $u_{\text {ac }}$ and recording the performance variable $z$ while the autopilot is in the loop. No external disturbances are assumed to be present during the identification procedure.

Example 19 (50\% control effectiveness). Consider $\lambda=0.50$. Figure 25 shows simulation results with the adaptive controller turned off: that is, autopilot-only control. Now, with the autopilot augmented by the adaptive controller, simulation results are shown in Fig. 26. After a transient, the augmented controllers provide better performance than the autopilot-only simulation.

Example 20 (25\% control effectiveness). Consider $\lambda=0.25$. With the adaptive controller turned off, that is, autopilot-only control, the simulation fails. With the autopilot augmented by the adaptive controller, simulation results are shown in Fig. 27. After a transient, the augmented controllers stabilize the system, whereas the autopilot-only simulation fails.

Figure 27 shows that the total control input $u$ reaches the actuator saturation level of $\pm 30 \mathrm{deg}$. To reduce the initial transient, we initialize the adaptive controller with the converged control gains $\theta$ from the 50\% control effectiveness case. As shown in Fig. 28, the initial transient is reduced as compared with initializing the control gains to zero. In this case, the actuator saturation level is not reached.

\section{Conclusions}

We presented the RCF adaptive control algorithm and demonstrated its effectiveness in handling non-minimum-phase zeros through numerical examples, illustrating the response of the algorithm under conditions of uncertainty in the relative degree and Markov parameters, measurement noise, and actuator and sensor saturations. Bursting was not observed in any of the simulations. We also suggested metrics that can serve as gain and phase margins for discrete-time adaptive systems. Future work includes the development of Lyapunov-based stability and robustness analysis of the RCF adaptive control algorithm as well as development of a theoretical foundation for analyzing broadband disturbance-rejection properties of the controller.

\section{Acknowledgments}

We wish to thank Rob Fuentes, Matthew Holzel, Matthew Fledderjohn, and Jesse Hoagg for helpful discussions.

\section{References}

[1] Narendra, K. S., and Annaswamy, A. M., Stable Adaptive Systems, Prentice-Hall, Englewood Cliffs, NJ, 1989.

[2] Goodwin, G. C., Ramadge, P. J., and Caines, P. E., "Discrete-Time Multivariable Adaptive Control," IEEE Transactions on Automatic Control, Vol. 25, No. 3, 1980, pp. 449-456.

doi:10.1109/TAC.1980.1102363
[3] Hoagg, J. B., Santillo, M. A., and Bernstein, D. S., "Discrete-Time Adaptive Command Following and Disturbance Rejection with Unknown Exogenous Dynamics," IEEE Transactions on Automatic Control, Vol. 53, No. 4, 2008, pp. 912-928. doi:10.1109/TAC.2008.920234

[4] Åström, K. J., Hagander, P., and Sternby, J., "Zeros of Sampled Systems," Automatica, Vol. 20, No. 1, 1984, pp. 31-38. doi:10.1016/0005-1098(84)90062-1

[5] Bai, E. W., and Sastry, S. S., "Persistency of Excitation, Sufficient Richness and Parameter Convergence in Discrete-Time Adaptive Control," Systems \& Control Letters, Vol. 6, No. 3, 1985, pp. 153-163. doi:10.1016/0167-6911(85)90035-0

[6] Rohrs, C., Valavani, L., Athans, M., and Stein, G., "Robustness of Continuous-Time Adaptive Control Algorithms in the Presence of Unmodeled Dynamics," IEEE Transactions on Automatic Control, Vol. 30, No. 9, 1985, pp. 881-889. doi:10.1109/TAC.1985.1104070

[7] Ioannou, P., and Sun, J., Robust Adaptive Control, Prentice-Hall, Englewood Cliffs, NJ, 1996.

[8] Ohkawa, F., and Yonezawa, Y., "A Discrete Model Reference Adaptive Control System for a Plant with Input Amplitude Constraints," International Journal of Control, Vol. 36, No. 5, 1982, pp. 747-753. doi:10.1080/00207178208932927

[9] Zhang, C., and Evans, R. J., "Amplitude Constrained Adaptive Control," International Journal of Control, Vol. 46, No. 1, 1987, pp. 53-64. doi:10.1080/00207178708933883

[10] Karason, S. P., and Annaswamy, A. M., "Adaptive Control in the Presence of Input Constraints," IEEE Transactions on Automatic Control, Vol. 39, No. 11, 1994, pp. 2325-2330. doi: $10.1109 / 9.333787$

[11] Lai, W. C., and Cook, P. A., "A Discrete-Time Universal Regulator," International Journal of Control, Vol. 62, No. 1, 1995, pp. 17-32. doi:10.1080/00207179508921532

[12] Lindquist, A., and Yakubovich, V. A., "Universal Regulators for Optimal Tracking in Discrete-Time Systems Affected by Harmonic Disturbances," IEEE Transactions on Automatic Control, Vol. 44, No. 9, 1999, pp. 1688-1704. doi: $10.1109 / 9.788535$

[13] Anderson, B. D. O., "Topical Problems of Adaptive Control," Proceedings of the European Control Conference, European Union Control Association, July 2007, pp. 4997-4998.

[14] Åström, K. J., "Direct Methods for Nonminimum Phase Systems," IEEE Conference on Decision and Control, Inst. of Electrical and Electronics Engineers, Piscataway, NJ, Dec. 1980, pp. 611-615.

[15] Johansson, R., "Multivariable Adaptive Control," Ph.D. Dissertation, Lund Inst. of Technology, Lund, Sweden, April 1983.

[16] Goodwin, G. C., and Sin, K. S., Adaptive Filtering, Prediction, and Control, Prentice-Hall, Englewood Cliffs, NJ, 1984.

[17] Praly, L., Hung, S. T., and Rhode, D. S., "Towards a Direct Adaptive Scheme for a Discrete Time Control of a Minimum Phase ContinuousTime System," IEEE Conference on Decision and Control, Inst. of Electrical and Electronics Engineers, Piscataway, NJ, Dec. 1985, pp. $1188-1191$.

[18] Johansson, R., "Parametric Models of Linear Multivariable Systems for Adaptive Control," IEEE Transactions on Automatic Control, Vol. 32, No. 4, 1987, pp. 303-313. doi:10.1109/TAC.1987.1104594

[19] Johansson, R., "Global Lyapunov Stability and Exponential Convergence of Direct Adaptive Control," International Journal of Control, Vol. 50, No. 3, 1989, pp. 859-869. doi: $10.1080 / 00207178908953402$

[20] Mareels, I., and Polderman, J. W., Adaptive Systems: An Introduction, Birkhäuser, Boston, 1996

[21] Hayakawa, T., Haddad, W. M., and Leonessa, A., "A Lyapunov-Based Adaptive Control Framework for Discrete-Time Nonlinear Systems with Exogenous Disturbances," International Journal of Control, Vol. 77, No. 3, 2004, pp. 250-263. doi: $10.1080 / 00207170310001649900$

[22] Akhtar, S., and Bernstein, D. S., "Lyapunov-Stable Discrete-Time Model Reference Adaptive Control," International Journal of Adaptive Control and Signal Processing, Vol. 19, No. 10, 2005, pp. 745-767. doi:10.1002/acs.876

[23] Bayard, D. S., "Extended Horizon Liftings for Stable Inversion of NonMinimum-Phase Systems," IEEE Transactions on Automatic Control, Vol. 39, No. 6, 1994, pp. 1333-1338. doi: $10.1109 / 9.293208$

[24] Venugopal, R., and Bernstein, D. S., "Adaptive Disturbance Rejection Using ARMARKOV/ Toeplitz Models," IEEE Transactions on Control Systems Technology, Vol. 8, No. 2, 2000, pp. 257-269. 
doi:10.1109/87.826797

[25] Hoagg, J. B., Santillo, M. A., and Bernstein, D. S., "Internal Model Control in the Shift and Delta Domains," IEEE Transactions on Automatic Control, Vol. 53, No. 4, 2008, pp. 1066-1072. doi:10.1109/TAC.2008.921526

[26] Santillo, M. A., and Bernstein, D. S., "A Retrospective Correction Filter for Discrete-Time Adaptive Control of Nonminimum Phase Systems," IEEE Conference on Decision and Control, Inst. of Electrical and Electronics Engineers, Piscataway, NJ, Dec. 2008, pp. 690-695.

[27] Santillo, M. A., Holzel, M. S., Hoagg, J. B., and Bernstein, D. S., "Adaptive Control of the NASA Generic Transport Model Using Retrospective Cost Optimization," AIAA Guidance, Navigation, and Control Conf., AIAA Paper 2009-5616, Chicago, Aug. 2009.

[28] Marsden, J. E., Basic Complex Analysis, W. H. Freeman, New York,
1973.

[29] Bernstein, D. S., Matrix Mathematics, 2nd ed., Princeton Univ. Press, Princeton, NJ, 2009.

[30] Juang, J. N., Applied System Identification, Prentice-Hall, Upper Saddle River, NJ, 1993.

[31] Landau, I. D., Adaptive Control: The Model Reference Approach, Marcel Dekker, New York, 1979.

[32] Åström, K. J., and Wittenmark, B., Adaptive Control, 2nd ed., AddisonWesley, Reading, MA, 1995.

[33] Tao, G., Adaptive Control Design and Analysis, Wiley, Hoboken, NJ, 2003.

[34] Mracek, C., and Ridgely, D., "Missile Longitudinal Autopilots: Connections Between Optimal Control and Classical Topologies," AIAA Paper 2005-6381, Aug. 2005.

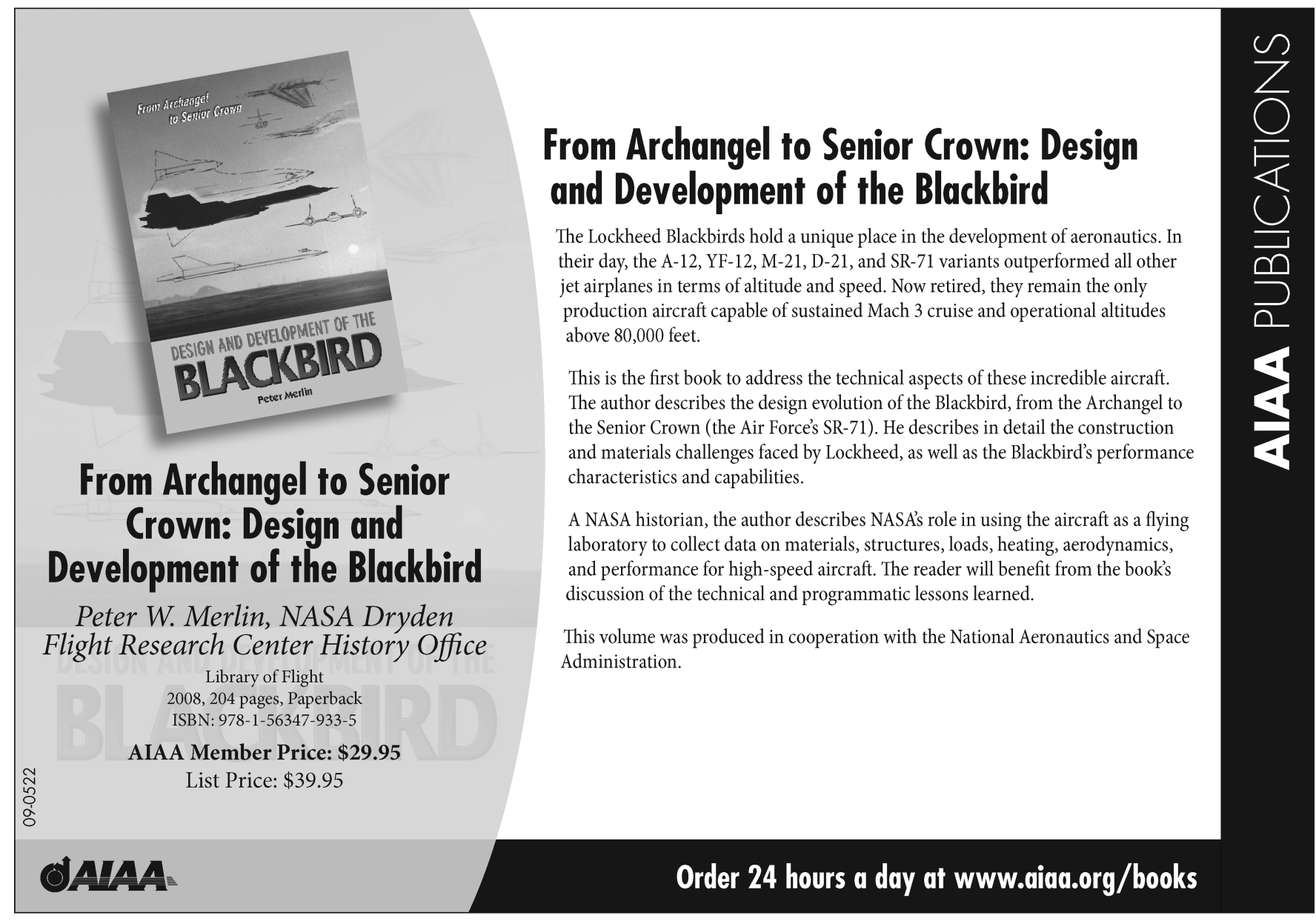

